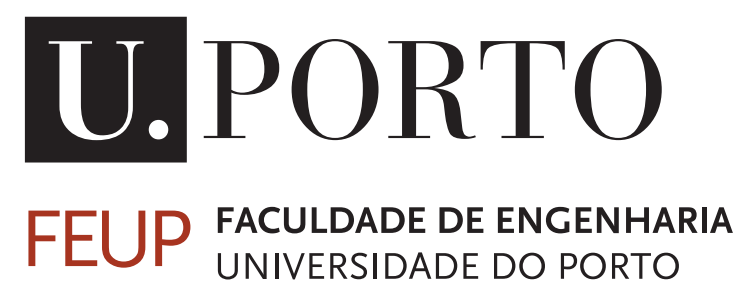

\title{
Visual Flight Rules-based Collision Avoidance System for VTOL UAV
}

\author{
Matheus Pedroso Sanches
}

\author{
Mestrado Integrado em Engenharia Eletrotécnica e de Computadores \\ Supervisor: Sérgio Reis Cunha $(\mathrm{PhD})$ \\ Co-Supervisor: Ricardo Faria (Altran Portugal)
}


(C) Matheus Pedroso Sanches, 2020 


\section{Resumo}

A utilização de UAVs, sigla em inglês para veículos aéreos não tripulados, na execução de tarefas complexas é cada vez mais frequente na atualidade. O setor de transportes privados passará em breve por uma grande mudança com a chegada de um novo conceito, os veículos elétricos de decolagem e aterragem vertical, conhecidos pela sigla em inglês eVTOL. A utilização desses veículos autónomos será feita, num primeiro momento, em conjunto com outras aeronaves tripuladas. Isso implica que aeronaves eVTOL terão que utilizar o atual espaço aéreo civil, no qual as autoridades de aviação exigem total conformidade com as regras que aplicam-se à Aviação Geral.

Nesse sentido, essa dissertação propõe um protótipo completo de um sistema anticolisão para aeronaves autónomas VTOL que encontrem-se a utilizar o espaço aéreo civil sob o efeito das chamadas Regras de Voo Visual (Visual Flight Rules). Para plena conformidade com as regulamentações existentes, o sistema anticolisão foi desenvolvido com base no estabelecido pelo Anexo 2 da Convenção sobre Aviação Civil Internacional, as chamadas Regras do Ar (Rules of the Air). A arquitetura proposta do sistema foi projetada para realizar diferentes tarefas, desde a deteção de tráfego pelos sensores, passando por averiguar se uma colisão é eminente ou não, até o início e término de uma manobra de evasão. Essas manobras encontram-se em conformidade com as regulamentações em vigor no que estabelecem para dois possíveis cenários de colisão entre aeronaves.

Todo o estudo consoante a esse sistema anticolisão foi por fim implementado e testado em microquadricópteros que têm a premissa de trazer resultados com valor experimental que possam conceder uma prova de conceito a todo o sistema desenvolvido. 


\section{Abstract}

The use of Unmanned Aerial Vehicles (UAVs) to perform complex tasks is getting more common today. The private transport sector will soon experience a significant change with the arrival of a new concept, the electric Vertical Take-Off and Landing (eVTOL) aircraft. These autonomous vehicles will, at first, be used in conjunction with other manned aircraft. That implies that the eVTOL aircraft will have to use the current civil airspace, where the aviation authorities require full compliance with the regulations that apply to General Aviation (GA).

In this sense, this dissertation proposes a complete prototype of a collision-avoidance system for VTOL UAVs using civil airspace under the regulations of the so-called Visual Flight Rules. To fully comply with existing rules, this collision avoidance system was developed based on what is established by Annex 2 to the Convention on International Civil Aviation, namely the Rules of the Air. The proposed architecture of the system was designed to carry out different tasks, from the detection of traffic by the sensors, through determining whether a collision is imminent or not, to the start and finish of an avoidance maneuver. These maneuvers comply with the regulations in what they establish for two possible aircraft collision scenarios.

The entire study corresponding to this collision avoidance system was implemented and tested in micro quadcopters that have the premise of bringing results with an experimental value that can grant proof of concept to the entire system developed. 


\section{Agradecimentos}

Muito tempo se passou desde que um rapaz de Sorocaba (São Paulo, Brasil), que tinha acabado de sair do Ensino Médio, decidiu ingressar no curso de Engenharia Elétrica da faculdade local. Aquela decisão foi o gatilho para uma série de acontecimentos que o levaram a sair do Brasil e começar uma nova jornada em Portugal, um país novo, desconhecido, mas que o recebeu de braços abertos. Todas as pessoas que o ajudaram, e as oportunidades que foram dadas a ele, são o motivo da existência desta secção de agradecimentos dessa dissertação de Mestrado. E é aqui que ele pretende mencionar e agradecer, por mais breve que seja, às pessoas que contribuíram para a sua graduação e para a conclusão desse trabalho.

Agradeço inicialmente a todos os professores que contribuíram para a minha formação, em especial ao meu orientador, Prof. Sérgio Reis Cunha, pelas valiosas ideias para esse trabalho e pelo apoio em momentos críticos. Agradeço também a toda a equipa da Altran Portugal pela proposta do tema e disponibilização do espaço e equipamentos utilizados nessa dissertação, em particular ao Eng. Ricardo Faria por ter sido sempre atencioso e por ter ajudado de todas as formas possíveis a solucionar os problemas que surgiram, sejam eles quais fossem.

Sou grato a todos os colegas de curso que me acompanharam e ajudaram durante toda a graduação nas três instituições de ensino pelas quais passei. No fim, todos ficamos a saber o quão desafiador, e gratificante, é concluir qualquer curso superior ligado à área de engenharia e tecnologia. Um agradecimento mais que especial aos meus amigos mais próximos que tive a alegria de conhecer, direta ou indiretamente, por conta destes anos de estudo. Todos eles, tendo conhecido no Brasil ou em Portugal, contribuíram para que esta jornada se tornasse mais fácil e leve. Os momentos que passamos juntos, e os que estão por vir, serão especiais para o resto da minha vida.

Por fim, e mais importante, sou grato a minha família por todo o amor e suporte que me tem dado ao longo de toda a vida. Sobretudo, agradeço aos meus pais Carlos e Alessandra por terem incentivado e apoiado todas as minhas decisões e pelo amor incondicional que têm pelos filhos. Tenho o orgulho máximo de eles serem meus pais. Sem os dois, nada disso seria possível.

Matheus Pedroso Sanches 
"Agir, eis a inteligência verdadeira. Serei o que quiser. Mas tenho que querer o que for.

$O$ êxito está em ter êxito, e não em ter condições de êxito. Condições de palácio tem qualquer terra larga, mas onde estará o palácio se o não fizerem ali?"

Fernando Pessoa em Livro do Desassossego 


\section{Contents}

1 Introduction 1

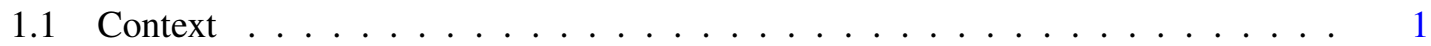

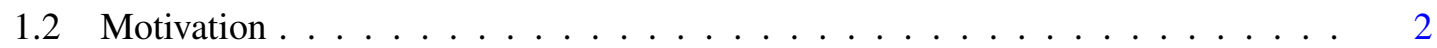

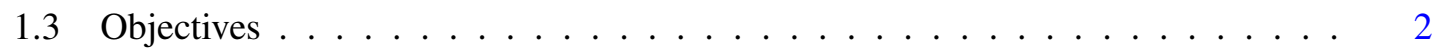

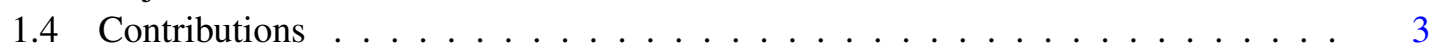

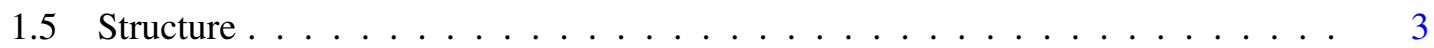

2 State of the Art 5

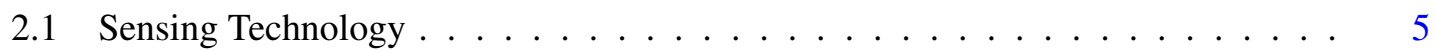

2.1.1 Non-cooperative traffic . . . . . . . . . . . . . . . . 5

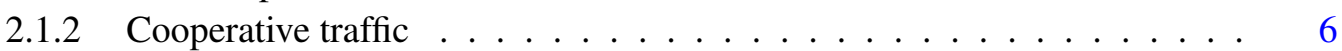

2.2 Collision Detection and Risk Evaluation Methods . . . . . . . . . . . . . . 8

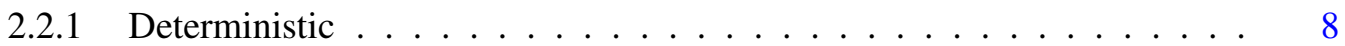

2.2 .2 Worst-case ......................... 9

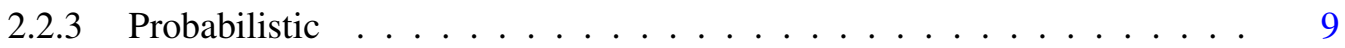

2.2 .4 Flight Plan Sharing . . . . . . . . . . . . . . . . . . . . 9

2.3 Collision Avoidance Methods . . . . . . . . . . . . . . . . . . . . . . . . . . 10

2.3.1 Geometric-based . . . . . . . . . . . . . . . . . . . 10

2.3.2 Potential Field . . . . . . . . . . . . . . . . . . . . . . 10

2.3.3 Sampling-based . . . . . . . . . . . . . . . . . . . 10

2.3.4 Cell Decomposition . . . . . . . . . . . . . . . . . . . . 11

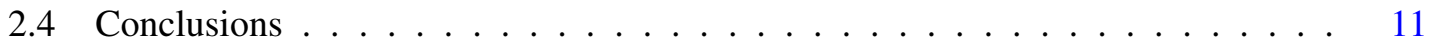

3 Systems Overview $\quad 13$

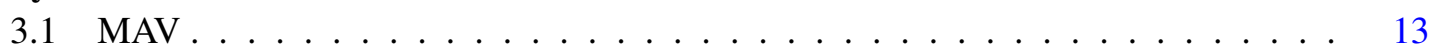

3.1 .1 Hardware . . . . . . . . . . . . . . . . . 13

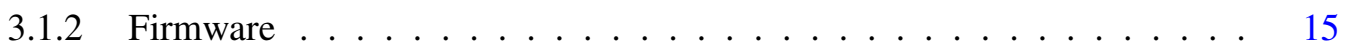

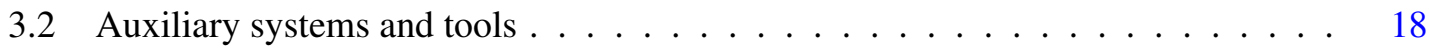

3.2.1 Indoor Positioning System . . . . . . . . . . . . . . . . . . . . 19

3.2 .2 Base station communication . . . . . . . . . . . . . . . . . 19

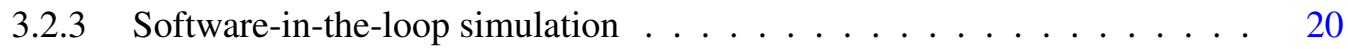

3.3 Conclusions . . . . . . . . . . . . . . . . . . . . 20

4 Implementation $\quad 23$

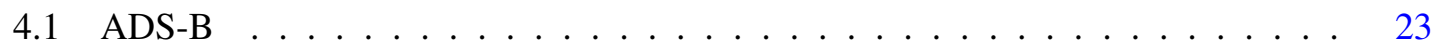

4.2 Conflict Detection . . . . . . . . . . . . . . . . . . . . 24

4.2.1 Tait-Bryan angles convention . . . . . . . . . . . . . . . 24

4.2 .2 System Modeling . . . . . . . . . . . . . . . . . . . . . 26 
4.3 Collision Avoidance Maneuver . . . . . . . . . . . . . . . . . . . . . . . . 28

$4.3 .1 \quad$ Visual Flight Rules . . . . . . . . . . . . . . . . . . . . . . . . . . . 29

4.3 .2 Evasive waypoint approach . . . . . . . . . . . . . . 30

4.3 .3 Potential field approach . . . . . . . . . . . . . . . . 31

5 Experiments and results 35

5.1 Flight simulator experiments . . . . . . . . . . . . . . . . 35

5.1.1 Artificial Potential Fields . . . . . . . . . . . . . . . . . . . 35

5.2 Real-world experiments . . . . . . . . . . . . . . . . . . . . 37

5.2.1 Peer-to-peer communication . . . . . . . . . . . . . . . . 37

5.2 .2 Head-on collision . . . . . . . . . . . . . . . . . . 38

5.2 .3 Close approach . . . . . . . . . . . . . . . . . 42

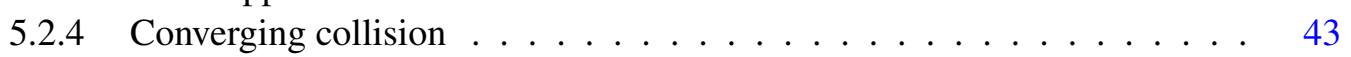

5.3 Conclusions . . . . . . . . . . . . . . . . . . . 46

6 Conclusions $\quad 49$

6.1 Future Work . . . . . . . . . . . . . . . . . . . 50

$\begin{array}{ll}\text { References } & 51\end{array}$ 


\section{List of Figures}

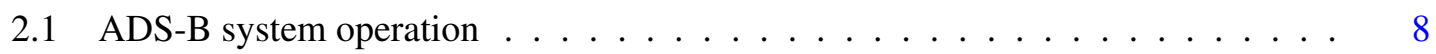

2.2 Approaches for collision prediction (a) Straight-line (Deterministic), (b) Worst Case, (c) Probabilistic and (d) Flight Plan Sharing . . . . . . . . . . . . . 9

3.1 System Hardware adapted from [28] - (a) Crazyflie 2.1, (b) Flow deck, (c) UWB Tag, (d) UWB Anchor, (e) Optical flow operation, (f) UWB localization . . . . . 15

3.2 Global architecture of the stabilizer module . . . . . . . . . . . . . . . . 16

3.3 Architecture of the Extended Kalman Filter module . . . . . . . . . . . . . . . 17

3.4 Architecture of the Cascade PID controller module . . . . . . . . . . . . . . 18

3.5 Loco Positioning System (a) Altran tests arena (b) Anchors layout adapted from [36] 19

4.1 Overall system architecture operation . . . . . . . . . . . . . . . . 24

4.2 Quadcopter body coordinate system and motor configuration . . . . . . . . . . 25

4.3 System modeling to determine $\tau(\vec{s}, \vec{v})$ (a) UAVs getting closer, (b) UAVs moving

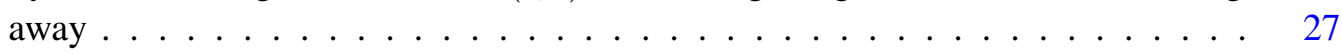

4.4 System modeling to determine $r_{\text {safe }}$ and $\vec{r}_{m} \ldots \ldots \ldots \ldots \ldots \ldots \ldots$

4.5 Collision avoidance in a converging traffic scenario . . . . . . . . . . . . . . 29

4.6 Collision avoidance in a head-on traffic scenario . . . . . . . . . . . . . 30

4.7 Scenario for the evasive waypoint $\vec{E}$ determination . . . . . . . . . . . . . . 31

4.8 Artificial potential field vector diagram . . . . . . . . . . . . 33

5.1 Position vs. time of the MAV without velocity error term . . . . . . . . . . 36

5.2 Position vs. time of the MAV with the velocity error term . . . . . . . . . . . 37

5.3 Initial detection and beginning of evasive maneuver of the head-on collision . . . 39

5.4 Evasive maneuver of the head-on collision scenario . . . . . . . . . . . . . . 39

5.5 Final trajectories and avoidance of head-on collision _ . . . . . . . . . . 40

5.6 Separation between the MAVs during the head-on collision scenario . . . . . . . 40

5.7 Collision detection analysis for head-on scenario . . . . . . . . . . . . . 41

5.8 Identification of head-on collision scenario . . . . . . . . . . . . . . . 41

5.9 MAVs inside each other safe zone in the close approach scenario . . . . . . . . . 42

5.10 Collision detection analysis for close approach scenario . . . . . . . . . . . 43

5.11 Initial detection and beginning of evasive maneuver of converging collision . . . 43

5.12 Evasive maneuver of the converging traffic collision scenario . . . . . . . . . . . 44

5.13 Final trajectories and avoidance of converging traffic collision . . . . . . . . . 44

5.14 Separation between the MAVs during the converging traffic collision scenario . . 45

5.15 Collision detection analysis for converging traffic scenario . . . . . . . . . 45

5.16 Identification of converging traffic collision scenario . . . . . . . . . . . . . 46 


\section{List of Tables}

5.1 Final values of scaling gains after fine-tuning $\ldots \ldots \ldots$. . . . . . . . . . 36

5.2 Average rate of received P2P packets with the same sending frequency . . . . . 38

5.3 Average rate of received P2P packets with different sending frequency $\ldots . . .38$ 


\section{Abbreviations}

ADS-B Automatic Dependent Surveillance-Broadcast

APF Artificial Potential Field

CPA Closest Point of Approach

CRTP Crazy Real-Time Protocol

EKF Extended Kalman Filter

eVTOL electric Vertical Take-Off and Landing

FAA Federal Aviation Administration

FEUP Faculdade de Engenharia da Universidade do Porto

GPS Global Positioning System

HLC High-Level Commander

ICAO International Civil Aviation Organization

IMU Inertial Measurement Unit

LiDAR Light Detection And Ranging

LOS Line of Sight

MAV Micro Aerial Vehicle

P2P Peer-to-peer

PC Personal Computer

PCM Protection Circuit Module

PID Proportional-Integral-Derivative

R\&D Research and Innovation

RF Radio Frequency

ROS Robot Operating System

SAA Sense and Avoid

SITL Software-In-The-Loop

SSR Secondary Surveillance Radar

ToF Time of Flight

UAM Urban Air Mobility

UAV Unmanned Aerial Vehicle

USB Universal Serial Bus

UWB Ultra-Wide Band

VMC Visual Meteorological Conditions

VFR Visual Flight Rules 



\section{Chapter 1}

\section{Introduction}

\subsection{Context}

With the increasingly common use of Unmanned Aerial Vehicles (UAVs), quadcopters particularly, drones are becoming very present in people's daily lives. The use of these vehicles has become so relevant to our society that a large bunch of areas is being affected by this "drone revolution". The use cases for safe, cost-effective solutions range from surveillance to delivery of packages passing by live entertainment and construction. The European Commission expects that, in the next 20 years, this market will employ more than 100 thousand people and have an economic impact that exceeds 10 billion euros per year, mainly in services [1].

While drones of today are used for such tasks as inspecting caves or power lines, larger models of tomorrow will transform industries like transportation. The electric Vertical Take-Off and Landing (eVTOL) aircraft are vehicles that promise to be an option for urban mobility within a few years [2]. It is an aerial vehicle with electric motors that is extremely quiet, thus being suitable for cities, which construction is very similar to a multirotor drone, but on a scale that makes it possible to transport passengers. Uber [3], Airbus [4], Boeing [5], and Rolls-Royce [6] are four of the big transportation companies that are working on the development of their autonomous flying drones for ferrying passengers around. The commitment of these and all other companies to autonomous driving will facilitate the use of these types of vehicles by people without specific aircraft pilot licenses, making them more suitable for private individual mobility than a helicopter, which needs a pilot.

Uber have already established missions and requirements for their eVTOL aircraft to enable aerial ridesharing in cities around the world. It is expected that initially, their vehicles will operate only in Visual Meteorological Conditions (VMC) [7]. VMC is an aviation flight category in which a pilot operates an aircraft in weather conditions clear enough to allow the pilot to see where the aircraft is going. That implies that these autonomous vehicles will have to adhere to some of the standards currently used in "recreational flying", namely the Visual Flight Rules (VFR). 


\subsection{Motivation}

Many efforts in research and development have been made so that these autonomous vehicles like eVTOL can be inserted into society safely and naturally. Altran is one of the companies that are trying to collaborate towards this safe integration. The market for the future Urban Air Mobility (UAM) awakened the interest of the company to make partnerships in the aerospace and automotive sectors to follow this new trend [8]. The Altran Portugal Research and Innovation (R\&D) team was assigned to strengthen these relations by developing solutions and systems that address the several challenges within the UAM domain. This work is motivated by that need, where one of the numerous technological fields is emphasized: the Sense and Avoid (SAA) capability of these vehicles.

The Federal Aviation Administration (FAA) considers SAA as "One of the key challenges to integrating UAVs into the National Airspace System" [9]. This term refers to the capacity of UAVs to detect and avoid collisions with other aircraft and obstacles during the flight. Conventionally, traffic collisions are avoided with commands from a control tower that gives instructions to the aircraft in the sky. This approach becomes difficult to implement regarding the number of vehicles that may be using airspace at the same time. This problem leads to the development of on-board mechanisms that guarantees an autonomous flight for the UAVs without any collisions.

The investment of big companies in the vanguard of this technology motivates the development of new approaches and algorithms that can guarantee a safe flight for this autonomous flying vehicles. However, while the standards and regulations of manned aviation are well established, the autonomous flight is an area that is still in development, which implies a careful approach by the regulatory associations. This new area is, in a lot of ways, similar to General Aviation (GA). This similarity promoted an extensive amount of work to study and understand the currently manned aviation regulations as a guide to aid in the development and design of SAA for unmanned aircraft [10]. In this dissertation, these regulations are explored to develop a complete functional collision avoidance system for VTOL UAVs based on standards and technologies commonly used in aeronautics.

\subsection{Objectives}

As aforementioned, there is a need to integrate UAVs into the existing manned airspace. This dissertation aims to adapt and implement a collision-avoidance system for the VTOL UAVs based on some flight safety concepts currently used by pilots in non-controlled airspace, the Visual Flight Rules (VFR).

Typically, a complete functional sense and avoid system is comprised of three main technological areas [10]: The sensors and trackers that sense the objects or traffic that the UAV is supposed to avoid. The collision detection methods that tells if the UAV is on a conflict trajectory or not. 
The collision avoidance algorithms that plan and execute the maneuvers to guarantee a collisionfree path. These topics are a guide for the development of this work. Each one of them envisions the following specific objectives:

- Study of the sensors and trackers that are used for surveillance inputs in a typical collision avoidance system, trying to adapt and reproduce its behaviors on a real-world scenario.

- Study of the methods used for collision detection of aircraft and modeling of a solution that can be used in a test arena taking into account the available hardware.

- Study of the algorithms used for collision avoidance maneuvers and implementation of them following the VFR, a concept that is defined by the International Civil Aviation Organization (ICAO) in the Rules of the Air standard [11].

This work is intended to study these technologies, models, and standards and implement them on a real-world quadcopter for proof of concept. All the implementation and tests of this system were made using micro quadcopters designed for research in the area of UAV control, which aims to bring the difficulties and challenges related to the use of real hardware in the design of these systems in practice.

\subsection{Contributions}

This work presents a complete prototype of a collision-avoidance system for VTOL UAVs operating in non-controlled airspace under Visual Flight Rules. The main contributions can be summarized as follows:

- Development of a Sense and Avoid architecture for collision avoidance system in VFR conditions. The proposed solution comply with the regulations established by the Rules of the Air standard.

- Construction and parameterization of collision avoidance maneuvers for two conflict scenarios defined by ICAO Rules of the Air: Head-on and Converging traffic scenarios.

- Development of a hybrid approach based on geometry and potential fields to generate collision avoidance maneuvers. As the construction and generation of these maneuvers are simplified, therefore the computational time is reduced.

- Development of a simple framework to detect cooperative traffic using a low-cost radio chip. The information provided by that framework is used to predict a possible collision scenario.

\subsection{Structure}

This dissertation contains five more chapters. In chapter 2, the State of the Art is described, and related works are presented. In chapter 3, some concepts regarding the architecture of the 
real-world system for experiments are exposed. Chapter 4 has the implementation details for the collision avoidance system. In chapter 5, the experiments and results are presented. Finally, in chapter 6 , the synthesis of the dissertation is made, and final considerations and perspectives for future research are presented. 


\section{Chapter 2}

\section{State of the Art}

This chapter shows the state of the art in which collision-avoidance systems for UAVs are. Section 2.1 reviews some of the sensors and technologies used in Sense and Avoid (SAA) systems. Section 2.2 presents a review of the approaches used to detect and evaluate the risks inherent to collisions. Section 2.3 reviews the algorithms used for evasion maneuvers of UAVs. Finally, section 2.4 makes some conclusions that guided the development of this work.

\subsection{Sensing Technology}

The technologies available for the UAV sensing capability can be categorized into two main approaches: Airborne technologies, e.g. sensors that are aboard the UAV, and Ground-based systems that are only capable to support the UAVs in a limited geographic and airspace region. According to Sahawneh [10] the airborne sensors are the best solution to provide a wide and dynamic SAA capability and are perfect for the real-time control systems that drive the vehicles in the sky autonomously.

There are quite examples of airborne sensors that are used to detect traffic and obstacles during the flight. Some technologies provide the detection of what is called cooperative and noncooperative traffic. The subsections that follow will bring a resume of each one of them.

\subsubsection{Non-cooperative traffic}

Non-cooperative traffic refers to any obstacle that does not possess systems that communicate their position, so the UAV has to find this traffic by itself. Some sensors like radar, optical/infrared cameras, and Light Detection And Ranging (LiDAR) are examples of technologies that detect this non-cooperative traffic.

\subsubsection{Radar}

Radar is one of the most used technology for the detection of air traffic for manned aircraft. It can also be used aboard the UAV, but the constraints of size, weight, and power consumption may 
result in some trade-offs between the radar range, bearing accuracy, and the angular field of view. According to Boskovic et al. [12], the long-range radars require a lot of power from the batteries for the higher transmit power. It is also harmful to the angular field of view if it is used a larger antenna with a higher directivity. The long-range also requires a narrower beam, which improves the bearing accuracy. However, this improvement reduces the field of view and requires additional antennas to be installed in the UAV.

The work of Mackie [13] showed that radar technologies have also problems with the strength of the return signal, giving that it is dependent on the material properties of the traffic that it is supposed to detect. This is harmful for the range estimation and could be a problem for UAVs that are encountering different types of traffic objects during the flight. This sensor has to be used with caution giving that it may not apply to all the UAV flights and navigation environments.

\subsubsection{Optical sensors}

Cameras and optical-flow sensors often appear in applications that require collision detection and avoidance. The resolution of the image and the processing power of the onboard controller are two of the most common factors that influence the performance of the system. The work of Lai et al. [14] put a high-resolution camera onboard a UAV and could detect traffic that was one kilometer away from it. However, cameras suffer from adverse weather and light conditions such as fog, clouds, precipitation, and sun glare, and other factors that can reduce the limit distance that an intruder can be detected.

\subsubsection{Infrared sensors}

The infrared sensors and cameras suffer from the same problems as the optical ones regarding the weather conditions but have the added advantage that they can be used at night. However, they both have difficulties in determining range and velocity in high-speed collision detection scenarios. LiDAR is a powerful technology that allows the determination of the distance from a laser emitter to an object or surface using a pulse laser beam, obtaining a 3D point cloud of the scene with notably high speed and precision. Zheng et al. [15] proposed a low-cost obstacle detection system based on a 2D LiDAR, which could detect obstacles in the environment during the movement of the UAV. Unfortunately, despite their decent results for static obstacles, robust LiDAR systems are generally too expensive and demand much power from the batteries, which can reduce the autonomy of the UAVs, and may not be suitable choices for all the applications.

\subsubsection{Cooperative traffic}

Alternatively, cooperative obstacles, that are equipped with transponders and systems such as the Secondary Surveillance Radar (SSR) and the Automatic Dependent Surveillance-Broadcast (ADSB), can transmit its current position, speed, and orientation status. These technologies make it possible for both aircraft to coordinate some action to avoid the conflict situation that can carry to a collision. 


\subsubsection{SSR}

The Secondary Surveillance Radar (SSR) system requires the aircraft to have specific equipment called a transponder, constituted by a device that is simultaneously receiver and emitter, which generates a response signal after interrogation from another aircraft or ground station. The interrogation is made by the radar antenna at the frequency $1030 \mathrm{MHz}$ and the response, originated aboard, at $1090 \mathrm{MHz}$ [16]. The response only carries the altitude of the aircraft, which is used in the decision process for a collision alert together with other data there are calculated afterward.

The SSR calculates the azimuth and distance of the targets based on the position of the antenna at the moment of emission of the impulse, the speed of propagation, and the delay time between the reception of the interrogation and transmission of the response [16]. This information is then processed by some security systems like the Traffic Collision Avoidance System (TCAS), which gives the corresponding collision alert to the pilot. Although the problems with size and transmission power from the common radar are not an issue here, this technology suffers from limitations on the line of sight and obstacles if it is intended to be used on UAVs that fly at lower altitudes [10].

\subsubsection{ADS-B}

Automatic Dependent Surveillance-Broadcast is by the definition of ICAO "A means by which aircraft, aerodrome vehicles, and other objects can automatically transmit and/or receive data such as identification, position, and additional data, as appropriate, in a broadcast mode via a data link." [17].

ADS-B is an extension of the SSR and works by transmitting regular position reports. The reports are sent without any request, received by receiving antennas on the ground (ADS-B OUT), and also by any aircraft in its range, provided that it is equipped with the necessary systems onboard (ADS-B IN). Cooperative information is shared over radio waves at $1090 \mathrm{MHz}$ or $978 \mathrm{MHz}$ on a broadcast mode, and it's relatively unaffected by adverse weather conditions. It relies on the principle to send many reports as possible, estimating that they will be captured by as many receivers on its range [16].

The usage of ADS-B, however, has its drawbacks. The technology is dependent on the Global Positioning System (GPS) that has good coverage of the airspace but has some areas where it becomes degraded, such as narrow valleys or urban canyons. ADS-B is also defective on the assumption that every other aircraft have the corresponding transponders for sharing data. That requires the widespread adoption of ADS-B technology to ensure detect-and-avoid reliability [10].

Research has been done in developing and testing ADS-B technology on UAV applications. Minucci et al. [18] proposed a method for exploiting the Wi-Fi modules already present in many small UAVs as a tool for sense and avoid adapting the ADS-B behavior. Despite the verified efficiency of their protocol, their experiments use a dedicated processor to encode information and send it to the other beacons. Y. Pan et al. [19] proposed a framework to control the total ADS-B communication traffic over the air. With a large number of flying UAVs using Automatic 


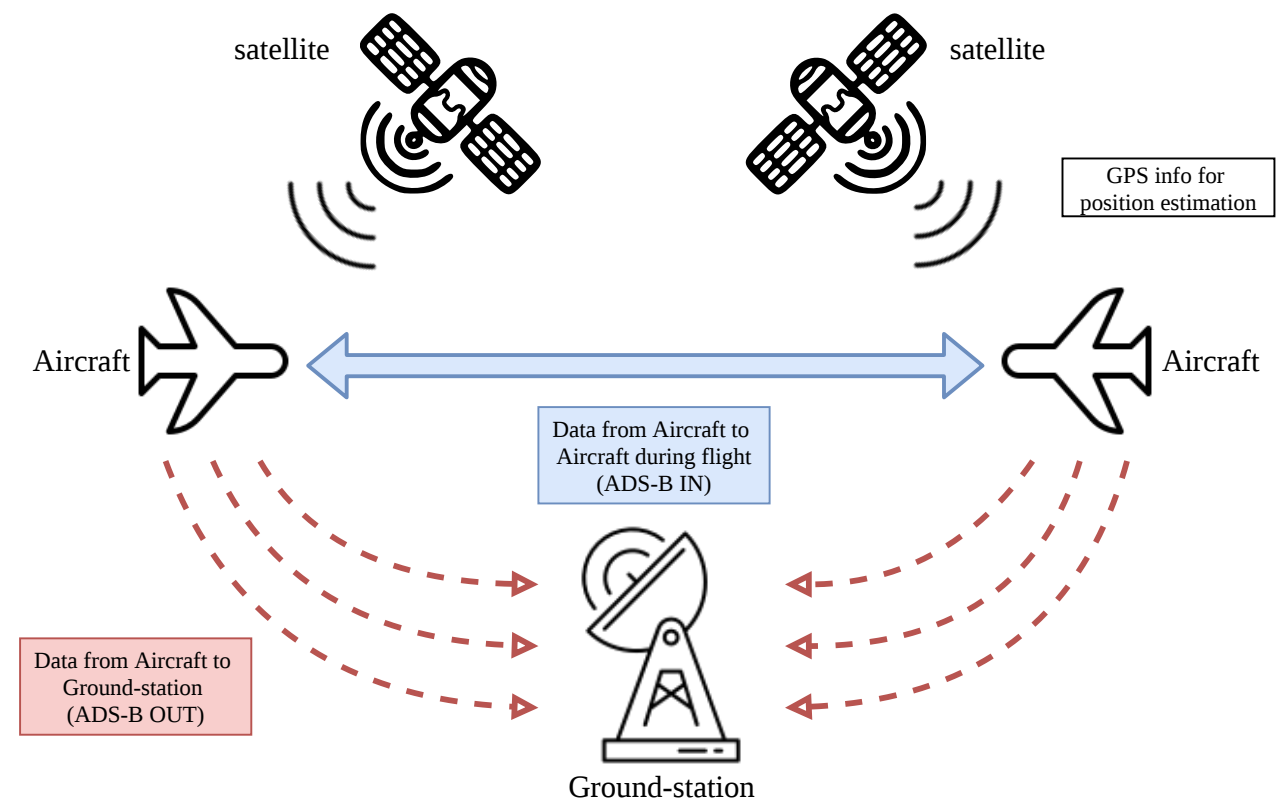

Figure 2.1: ADS-B system operation

Dependent Surveillance-Broadcast, the ADS-B communication may be interfered, which could result in aerial collisions. That framework tries to effectively schedule the UAVs' flights over a specific area without interfering with the communication. Their solution proved to be effective with generated trajectories based on an airplane dataset, but further tests have to be conducted in real-world experiments.

\subsection{Collision Detection and Risk Evaluation Methods}

The collision detection problem is one of the most challenging areas for the SAA field. In this concept, a UAV has to estimate the position of conflict traffic and apply some predefined metrics, such as time, distance, and cost on the estimated scenario to decide whatever or not a potential collision exists. The source of errors that came from this estimation, like noise and modeling of the dynamics of the intruder, make the risk evaluation methods crucial to detect collisions without creating too many false alarms. Albaker \& Rahim [20] classified the different methods under four fundamental approaches: deterministic or straight line, worst-case, probabilistic, and flight plan sharing.

\subsubsection{Deterministic}

The straight-line approach is the simplest and is used in the majority of the applications. It consists of predicting a single trajectory of the intruder, without consideration of any sensor or model uncertainties, using a single line extrapolation. It's a satisfactory approach when the trajectories of 


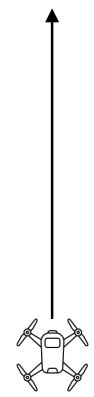

a)

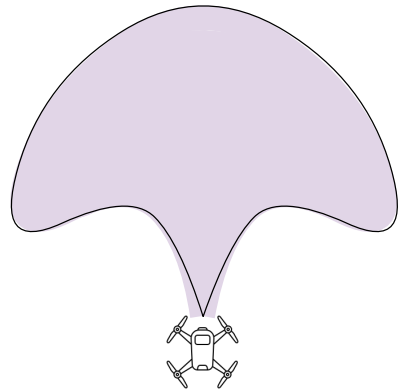

b)

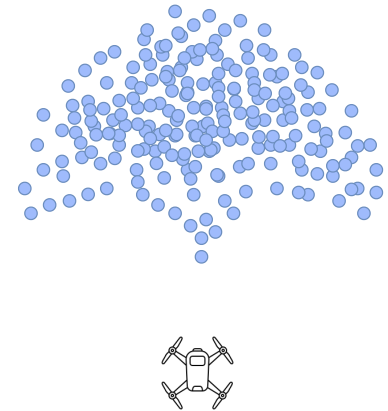

c)

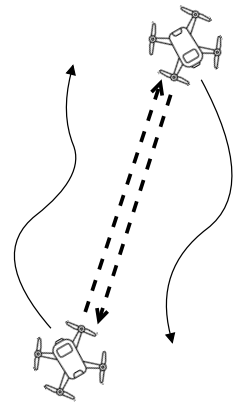

d)

Figure 2.2: Approaches for collision prediction (a) Straight-line (Deterministic), (b) Worst Case, (c) Probabilistic and (d) Flight Plan Sharing

the encountering aircraft are very predictable in a short time. However, in practice, the uncertaintyfree model could lead to an erroneous prediction of the collision thread [20].

\subsubsection{Worst-case}

Assuming that the conflicting traffic can perform any maneuvers, the worst-case approach is used to predict any trajectories that could cause a collision between both aircraft. This is beneficial in a way that can inhibit the problem with the free model of the deterministic approach, but it is quite processing demanding, which implies that the collision scenarios have to be predicted in a short period projection. It is also the approach that most leads the system to false collision alarms [20].

\subsubsection{Probabilistic}

Probabilistic methods estimate the probability of a collision based on a model of future intruder dynamics. These probabilities are often estimated by an analytical solution, numerical approximation, or Monte Carlo methods [10]. These estimations are then compared to a certain threshold and the system provides if a collision is imminent or not. The probabilistic approach provides a balance between relying on either a single-trajectory model or a set of worst-case maneuvers. The work of Owen et al. [21] had included in their collision risk evaluation module the effects of both dynamic uncertainty (the possibility that aircraft may maneuver unexpectedly) and surveillance uncertainty (due to noisy sensor data). To address both forms of uncertainty, they consider the collision detection problem as a Markov Decision Process (MDP).

\subsubsection{Flight Plan Sharing}

These methods rely on the assumption that the conflict object communicates its trajectory path and specific information (position, heading, and velocity) to all the other aircraft in the vicinity. This gives the UAV a full picture of its neighboring aircraft movements, which may lead to a precise projection of the encounters states. This type of collision detection can be achieved with the use 
of technologies as the ADS-B. Albaker \& Rahim [22] proposed a direct and efficient method of collision detection and avoidance for cooperative unmanned aircraft systems based on negotiation and predefined maneuvers.

\subsection{Collision Avoidance Methods}

After a collision scenario is detected, the system must select a proper action to be performed to evade the conflict object. That are two main approaches to compute and execute this proper action: Deliberative and Reactive motion planning. The first one relies on the computation of maneuvers based on a priori knowledge of the environment, and the latter uses a behavioral approach to react to on-board sensor information [10].

The most common collision avoidance methods are geometric-based guidance methods, potential field methods, sampling-based methods, and cell decomposition techniques.

\subsubsection{Geometric-based}

The geometric approach is the most simple method to compute the collision maneuver. It is intuitive as it relies on fast analytical solutions based on the kinematics of the UAV and in the geometry of the collision scenario. This approach usually assumes a straight-line projection to determine if the conflict object will penetrate in the UAV circular safe zone giving its current speed. The work of Alturbeh \& Whidborne [23] proposed a Decision-Making System (DMS) where the avoidance maneuvers are parameterized using a geometric approach for aircraft that are flying in civil airspace under Visual Flight Rules. Their collision avoidance approach is also based on the Rules of the Air standard. However, their framework is focused only on fixed-wing aircraft, and studies with other types of UAVs are needed. Moreover, they only simulated the proposed algorithms, and real-world implementation is desired.

\subsubsection{Potential Field}

This approach is another one that is heavily used for collision avoidance in robotics. This potential field actuates as a virtual force on the UAV usually as attractive from the goal and repelling from nearby traffic. This method is usually very efficient giving that, for each moment, the motion of the robot is determined by the potential field at its location. Budiyanto et al. [24] developed an optimal path planning for a quadcopter model in Gazebo Simulator using this method. They have succeeded in the collision avoidance for static and dynamic obstacles considering the dynamics and kinematic equations of the UAV on their model. Still, this strategy is subject to local minima, where the robot can get trapped, and heuristic developments to escape these points are needed.

\subsubsection{Sampling-based}

Sampling-based methods like the Probability Road Maps (PRM) and Rapidly-exploring Random Trees (RRT) are good ways to produce collision-free motion and path planning, especially for 
ground robots. They usually consist of two phases: construction and a query phase. In the construction, the algorithm tries to efficiently search nonconvex, high-dimensional spaces by randomly building a space-filling graph, approximating the motions that can be made in the environment. In the query phase, the start and goal configurations are connected to the graph, and the path is obtained by some path planning algorithm like Dijkstra's shortest path query.

These methods usually require significant computation time for the re-planing of the trajectories which makes then difficult to implement for reactive collision avoidance. However, some works, like the one from Lin \& Saripalli [25], showed that good results can be achieved with this method if some extensions are made for the original algorithm. Using a simplified node connection strategy and using a reachable set instead of the single nominal point assumption to predict the obstacle's motion, this approach was able to find a feasible motion plan in a relatively short time with probabilistic completeness.

\subsubsection{Cell Decomposition}

The basic idea behind the cell decomposition technique is to subdivide the free space of the UAV's configuration into smaller regions called cells. After this decomposition, a connectivity graph is constructed according to the adjacency relationships between the cells, where nodes are the free space and the links show that the corresponding cells are adjacent to each other. From this constructed graph, a continuous path is determined by simply following adjacent free cells from the initial point to the goal point. Generally, cell decomposition methods are considered to be global path planner that requires a priori knowledge of the environment [10].

The work of Shanmugavel et al. [26] showed that this approach is useful when used for collision avoidance on static obstacles like buildings. Still, for moving obstacles, like another $\mathrm{UAV}$, this approach suffers from the same problems as the sampling-based methods giving the extensive computation time for the re-planing.

\subsection{Conclusions}

From the content exposed throughout the previous sections of this chapter, it is possible to withdraw key conclusions to guide this dissertation.

- One way to find the traffic to be avoided is to develop means by which the UAV can automatically transmit and/or receive data related to the position, speed, and orientation with other vehicles that share the same airspace. Such functionality can be achieved by adopting technology like the Automatic Dependent Surveillance-Broadcast (ADS-B) to the system.

- The collision detection can use the information provided by the ADS-B and a kinematic model of the aircraft to project the movement of the detected intruder forward in time to identify possible conflicts or collisions. 
- The Flight Plan Sharing approach can be a powerful method to detect a collision between cooperative traffic. It has a balance between processing power and a good prediction of collision scenarios without many false alarms.

- The Geometric-based and the Artificial Potential Field are the approaches that implement reactive motion planning. Giving that a priori knowledge is not always available, researchers tend to use the reactive approach to conceive collision avoidance algorithms for dynamic scenarios and where the time window for the avoidance maneuver is short. 


\section{Chapter 3}

\section{Systems Overview}

This chapter presents the entire architecture of the systems used for the design and experiments of this dissertation. Section 3.1 presents the micro quadcopters and its modules that were used for the collision avoidance methods. In section 3.2 the auxiliary systems and the software tools used in real-world and simulation tests are presented. Finally, section 3.3 make some conclusions about the key points that guided the implementation part.

\subsection{MAV}

The UAVs used in this work for the real-world experiments were the Crazyflie 2.1 by the Swedish company Bitcraze [27]. It is a micro quadcopter designed to be an open-source flying development platform. This Micro Aerial Vehicle (MAV) is a small quadcopter with plastic propellers that only sizes $130 \mathrm{~mm}$ and weighs $27 \mathrm{~g}$. Its size is ideal for flying indoors, as it could be easily studied flying from up close.

The project gives complete control over the hardware, the firmware running on the quadcopter as well as the client library that runs off-board. Bitcraze hosts all of its codes on their GitHub repository [28].

Some important aspects of this quadcopter regarding its hardware and firmware were important to the development of this work and will be listed below.

\subsubsection{Hardware}

The quadcopter is divided into two main chips, the nRF51822 for the radio communications and the STM32F405 microcontroller for all the processing to control the quadcopter.

The battery used by the Crazyflie is a Lithium-Polymer (LiPo) battery that supplies 3.7V and has a capacity of $250 \mathrm{mAh}$. The battery's discharge rate is $15 \mathrm{C}$, which in theory provides to the quadcopter an autonomy of 4 minutes of continuous flight. The battery also comes with a Protection Circuit Module (PCM) attached to it that prevents the user from under or overcharging the battery or from shorting it. 
The quadcopter is equipped with four brushed DC motors that are coreless, which provides faster acceleration. However, the motors require more torque at higher speeds to fight the increased air resistance and therefore more current from the battery is needed. Attached to the four motors are plastic propellers that are fragile, but mitigate possible damages in case of accidents [29].

Finally, the quadcopter has some sensors there are built-in on the main Printed Circuit Board (PCB) and others that are available by the purchase of expansion decks. The PCB has a high precision Inertial Measurement Unit (IMU) with a 3-axis accelerometer/gyroscope (BMI088), for the measurement of acceleration in body-fixed coordinates and angle rate in roll, pitch, and yaw, and a pressure sensor (BMP388) that measure relative vertical position. The sensors available by the expansion decks are the optical flow (PMW3901) that measures the detection movement of pixels, a laser Time-of-Flight (ToF) sensor (VL53L1x) for the distance to a surface, and an Ultra Wide Band (UWB) sensor (DWM1000) that measures the distance between two UWB modules.

\subsubsection{Optical flow sensor}

The term optical flow refers to a flow of two-dimensional images, in which certain features, such as patterns or pixel intensities, are tracked in time. This sensor is a downward-facing camera that is positioned below the quadcopter with the purpose to "see" how the pixels patterns on the ground are moving. This tracking pattern makes the operation of this sensor over a blank surface very difficult. With the difference in pixels between each frame and the current height, the optical flow deck can give the velocity that the quadcopter is moving in the XY plane [30].

\subsubsection{ToF laser sensor}

This is a downward-facing laser emitter and receiver that is mounted together with the optical flow sensor to give the current height of the quadcopter. When estimating height using ToF, the distance is just the product between the time that the emitted pulse takes to travel from the MAV, reflect on the ground and go back to the receiver, and the signal speed, which depends on the technology used. This sensor provides centimeter precision measurements depending on the sample rate, mode of operation, and the properties of the reflective surface [30].

\subsubsection{UWB sensor}

The Ultra-Wide Band (UWB) sensor is the receiver module (Tag) of the indoor positioning system. It works by receiving messages from the nodes (Anchors) that are in a known position. With the measurement of the running time of flight between the MAV and one anchor, the distance between then is calculated. Then the distances from many anchors are used to estimate the absolute position of the quadcopter in the test arena. More of this system and its relevance to the work are presented in section 3.2. 


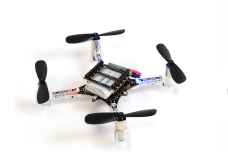

a)

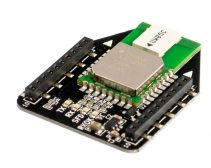

c)

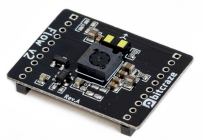

b)

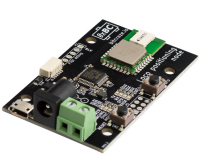

$d)$

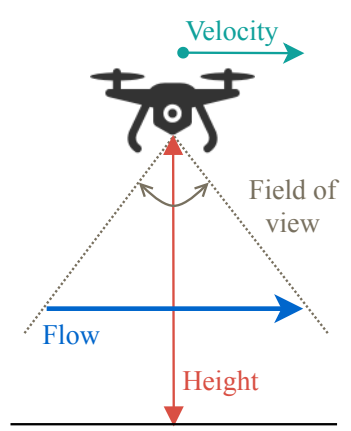

e)

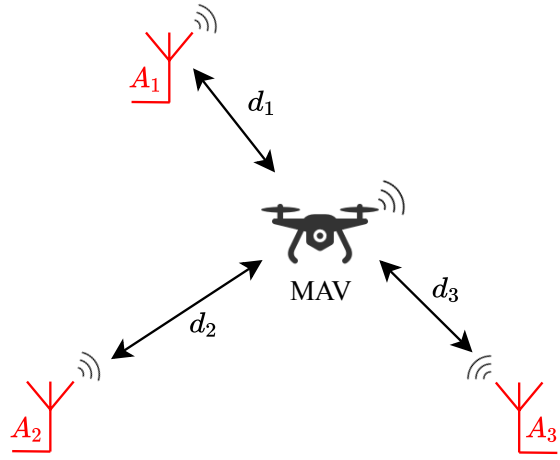

f)

Figure 3.1: System Hardware adapted from [28] - (a) Crazyflie 2.1, (b) Flow deck, (c) UWB Tag, (d) UWB Anchor, (e) Optical flow operation, (f) UWB localization

\subsubsection{Firmware}

The Crazyflie firmware is based on the Free Real-Time Operating System (FreeRTOS), an opensource operating system. It handles the scheduling of processes and controls the flight calculations. The firmware is written in $\mathrm{C}$ and is composed of several source code and headers, structuring the code in different layers of abstraction. The first layers (drivers and hal) handle the hardware and built-in sensors. The deck layer covers the interfacing of extension decks like the Flow deck and the UWB positioning deck. Finally, all the operations (stabilizer loop) are done in the highlevel functions of the module layer [31]. The module layer is the one studied in detail in the implementation chapter. Some of its functionalities that control the quadcopter will be presented below.

\subsubsection{Stabilizer}

The small size of the Crazyflie offers several challenges in adopting it as a research platform. Its small inertia requires controllers that can react with very little latency, or else it won't fly properly. To be the most efficient possible, all the calculations necessary to stabilize the quadcopter is performed by its firmware [29].

The stabilizer is composed of basically three modules, and the operation of them depends on the type of stabilization wanted. The first one is the state estimator that gives the absolute position, velocity, and orientation of the quadcopter. Then comes the commander part, which receives the setpoints by radio from the client application, processes it, and gives the position to go. Finally, the controller, given the current state and the desired setpoint, calculates how much thrust the quadcopter needs to reach the position wanted. Figure 3.2 shows a diagram of the aforementioned modules. 


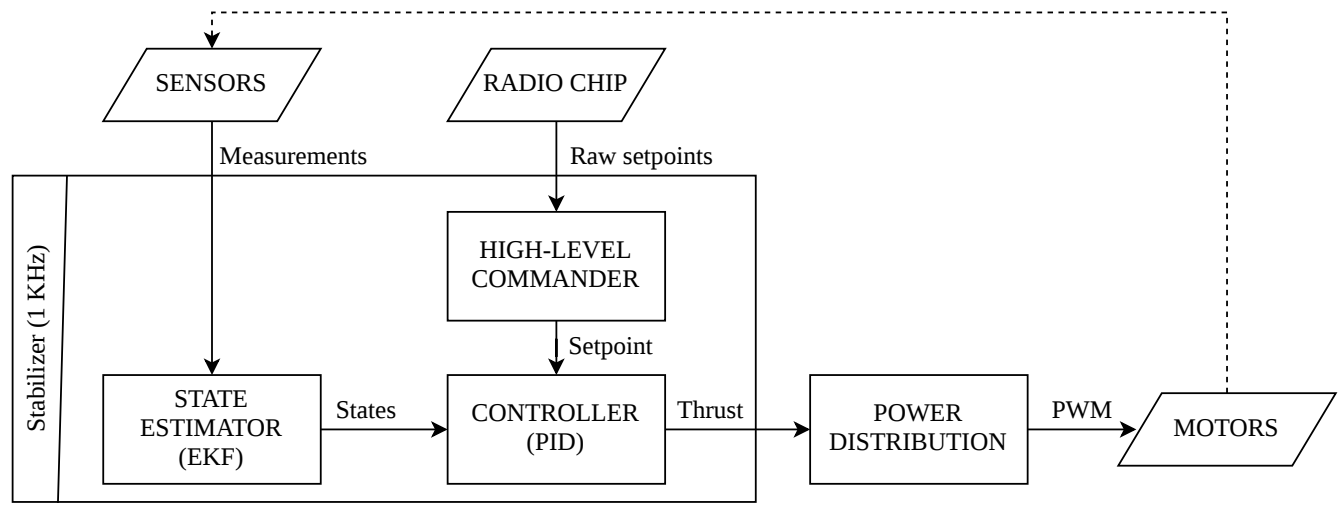

Figure 3.2: Global architecture of the stabilizer module

\subsubsection{State Estimator}

State estimation is really important in robotics, given that for most applications is necessary to track some information about the current state of the robot. This module has the role to interpret the measurements that come from different sensor sources, fuse them, and estimate the current position, velocity, and orientation of the robot. This is vital information for autonomous systems that wants to guarantee a reliable motion [29].

There are different methods for state estimation. Nevertheless, for a quadcopter with the type of sensors presented in the previous section, and with the main chip processing power, some are preferred than others. The Crazyflie source code contains both a complementary filter and an Extended Kalman Filter (EKF). The EKF is the only one that estimates all the states necessary for the collision avoidance algorithms, and that is why is the one used along within this work.

The EKF is a recursive filter for non-linear systems that estimates the current state of the quadcopter based on all past measurements. It uses the measurements from the several sensors (in combination with a predicted standard deviation of the noise that comes with these measurements) and the dynamic mathematical model of the system itself to give the best estimation of its current position, velocity, and orientation. The filter is organized under the following steps that are repeated at each loop:

- The prediction: At a certain time, the current estimated position is known, as well as the dynamic model. From that, the next position is predicted, which becomes the new estimated position.

- The correction (or update): The estimated position needs to be corrected to correspond more to the real position. The measurements are then interpreted to update the estimated state.

This work is not intended to go into detail on the theoretical part of the EKFs, but, if the reader wants, further information can be found on [32]. 


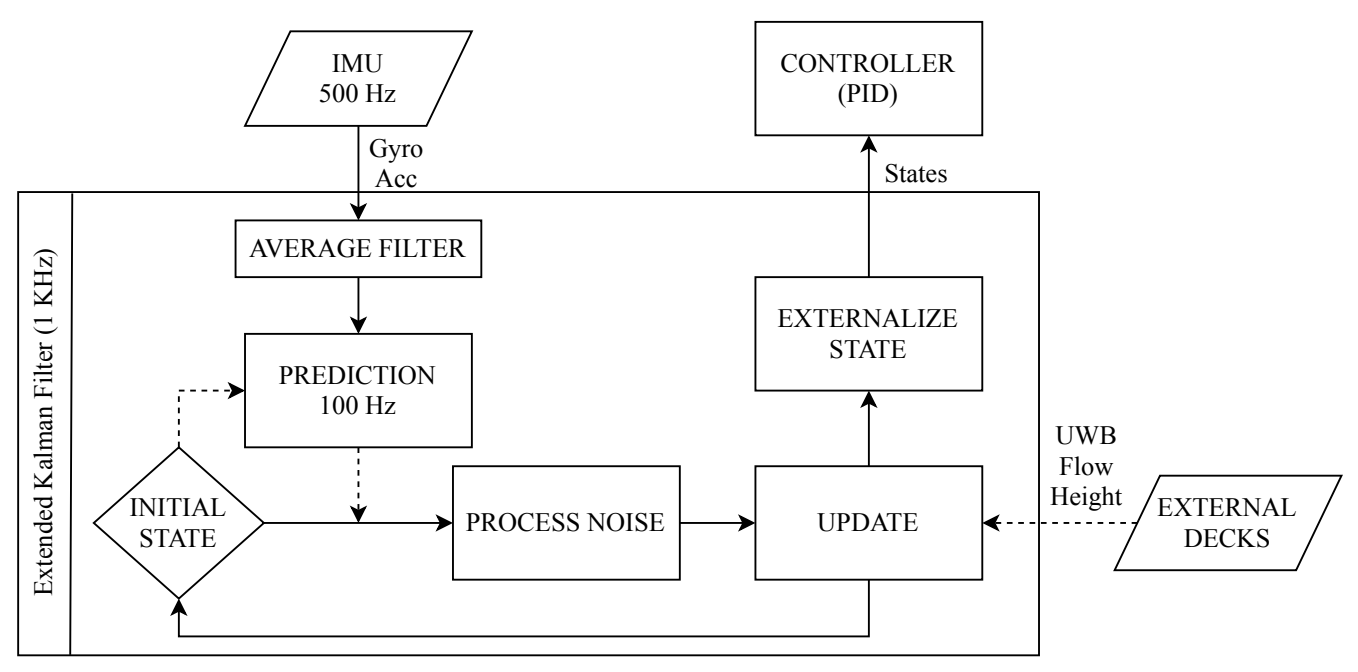

Figure 3.3: Architecture of the Extended Kalman Filter module

\subsubsection{Commander}

The commander module has the role to give a position to go, with absolute or relative positioning. It receives the setpoint from the client application by radio and tries to process it with two possible methods. The first one only gives to the quadcopter the final position that it is supposed to go without any intermediate points. This method has the drawback that if a setpoint is too far from the current position, the regulation could move the quadcopter with an extreme command, and could eventually lead to flips, huge oscillations, or crashes. Most regulation algorithms choose to avoid this problem by creating intervals of values that cannot be exceeded. However, it seems better to create intermediary setpoints to smooth the trajectory. A high-level commander (HLC) is implemented in the firmware to do so.

The HLC handles the raw setpoints from the radio and generates a predefined trajectory to it. The high-level commander uses a planner to generate smooth trajectories with 7 th order polynomials. The planner generates a group of intermediate setpoints, which will be handled by the HLC and send one by one to the commander framework. More details about this implementation can be found in the work of Preiss et al. [33].

\subsubsection{Controller}

Once the current and the desired positions are known, the quadcopter has to move appropriately. Indeed, moving too fast could lead to dangerous behaviors (eg. flips and crashes). On the opposite, a slow movement could lead to drifts and low reactivity. The Crazyflie hosts three different control algorithms. The most used Proportional-Integral-Derivative controller (PID), the Mellinger Controller [34], and the Incremental Nonlinear Dynamic Inversion (INDI) controller [35]. The last two are suitable for precise position estimate (Motion Capture Systems), and given that this work does not have access to these technologies, the PID is preferred. 


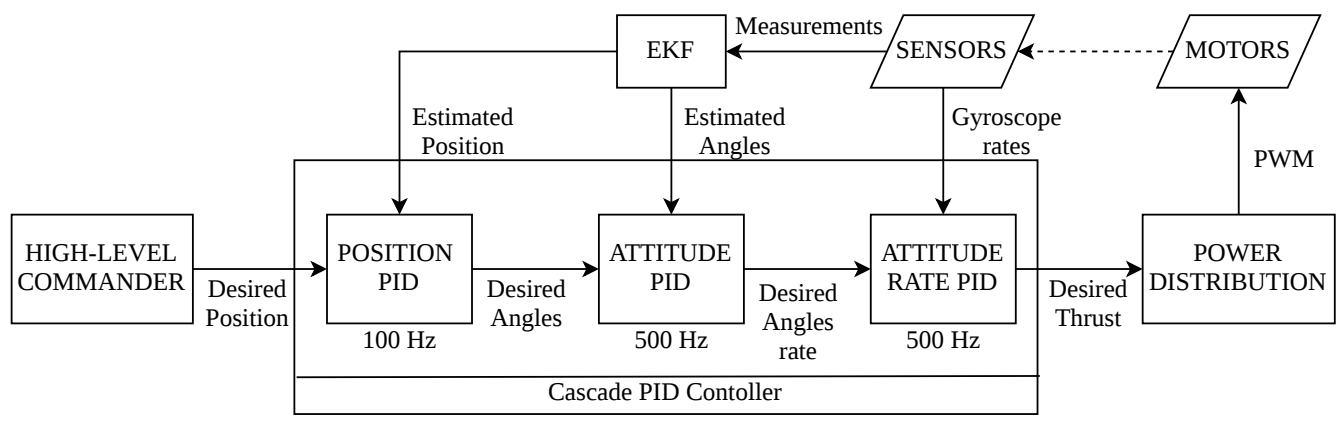

Figure 3.4: Architecture of the Cascade PID controller module

A PID controller calculates an error value from the difference between the desired setpoint and a measured variable at each control loop. It then applies a correction based on the sum of a proportional (P), an integral (I), and a derivative (D) terms. Each term is adjustable with coefficients as each of the latter represents a different aspect of regulation [29].

- The proportional term is the current error value. The further the desired value is, the bigger this term will be.

- The integral term is the sum of error values over time. It is proportional to both the magnitude of the error and the duration of the error.

- The derivative term is the slope of the error over time. The strongest the slope is, the bigger this term will be.

This framework uses the desired position provided by the commander to feed a system composed of three PID controllers, each of them controlling a different aspect of the quadcopter. The desired position enters on a Position PID that converts it to desired pitch and roll angles. These are injected in the Attitude PID that determines the desired angles rates which are sent to the angle rate controller. After processing, the Attitude Rate PID gives the desired thrust that is sent to the motors by the power distribution interface.

\subsection{Auxiliary systems and tools}

Some software and hardware tools were used to assist in the development of this dissertation. These systems made possible that tests with the proposed methods and algorithms could be done in a controlled indoor environment. The software tools also aid in obtaining the results from the several real-world experiments, and with the tests that were conducted on a flight simulator that worked as a first validation of the developed collision avoidance methods. A detailed description of the aspects of these systems is presented below. 


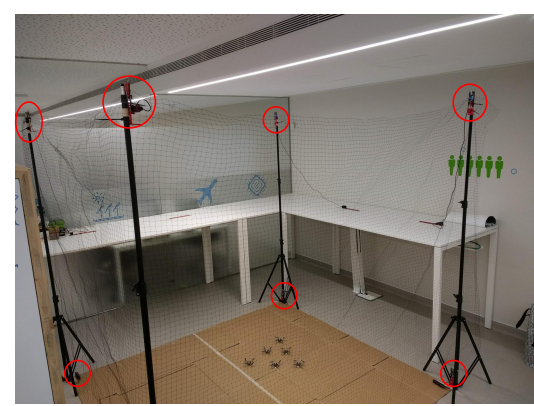

а)

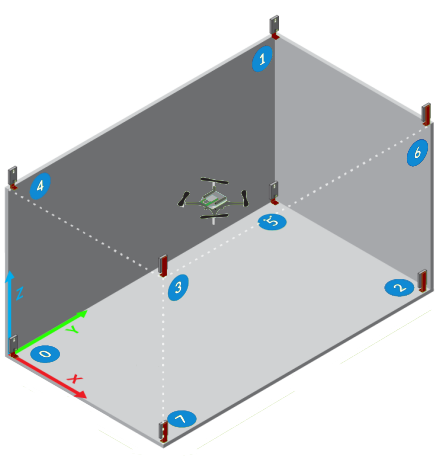

b)

Figure 3.5: Loco Positioning System (a) Altran tests arena (b) Anchors layout adapted from [36]

\subsubsection{Indoor Positioning System}

The Loco Positioning system is used to find the absolute 3D position of the quadcopters in space. It is in many ways similar to a miniature GPS system. The system uses a set of "Anchors" (like GPS satellites) that are positioned in the room to be the absolute reference to the receiver modules (tags) that are coupled on the quadcopters [36].

By sending short high-frequency Ultra-Wide Band (UWB) radio messages between the receiver and the anchors, the system measures the distance from each reference to the quadcopter and calculates its position from that information. For the exact localization of the MAV at least 3 receivers are necessary (trilateration). In contrast to Bluetooth Low Energy and Wi-Fi, positioning is done with Time of Flight (ToF) methodology instead of the measurement of the Receive Signal Strength Indicator (RSSI). It is based on the Decawave DWM1000 chip and has an accuracy in the $10 \mathrm{~cm}$ range. The $3 \mathrm{D}$ positioning performance depends on the system setup and environment as the line-of-sight between receivers and transmitters has a big impact on the accuracy.

As represented in figure 3.5, this indoor positioning system was mounted in Altran's office to be used in the experiments of chapter 5. This system provides the main source of position reference inside the test arena for the MAVs used in this work. Although the accuracy of estimation is lower compared to the more advanced Motion Capture Systems (MOCAP), this open-source tool was enough to be the ground truth of the experiments with a lower price and a simple and portable setup.

\subsubsection{Base station communication}

All communication between the computer and the quadcopters is done through the Crazyflie Python Library, available as open-source software through the Bitcraze GitHub account [37]. The library contains all the necessary structures to establish with the PC an individual connection with each Crazyflie through a radio dongle over $2.4 \mathrm{GHz}$ or by USB cable. This framework is used as 
the interface to send position setpoints to the quadcopters that are involved in the collision avoidance scenarios. It is used to send a position that will result in a collision, so the onboard collision avoidance algorithms can sense and avoid the other quadcopters.

This tool also retrieves the relevant data (id, position, velocity) from the quadcopters in realtime. As the quadcopters do not have enough memory to store the evolution of these variables for the whole flight of the experiments, this framework was used to get the data for the results of chapter 5 .

\subsubsection{Software-in-the-loop simulation}

In a first test approach to the implemented collision avoidance algorithms, a flight simulator based on the Gazebo simulation environment was used. This simulator is a work of the Crazyflie community that uses a Gazebo model of the quadcopter from the manufacturer Bitcraze [38], physical parameters, i.e inertia, motor parameters, and drag coefficients, from Föster's work [39], and motor and sensor models from RotosS framework [40].

This open-source project provides support for both software-in-the-loop (SITL) and hardwarein-the-loop (HITL) simulation of the Crazyflie. To accomplish a more complete simulation, the project simulates the sensor data by using some information gathered from Allan Standard Deviation analysis of the quadcopter's real sensors. This project provided a tool to simulate all the algorithms and modifications embedded before testing it on the real quadcopters with minimum modification (no changes in the overall behavior) of the original firmware code. The source code and further information can be found in [41].

\subsection{Conclusions}

The exposed technologies and tools provide some insights into what can be done for the implementation of the proposed collision avoidance system.

- The currently available hardware of the quadcopters does not dispose of sensors that provide the detection of non-cooperative traffic. This limitation implied the focus of this dissertation was in the tracking of cooperative traffic only.

- The radio communication of the quadcopter could be extended to send broadcast messages for all the other MAVs in the proximity. The state estimator module could provide position and velocity estimations to be sent in these messages. These two approaches can be used to simulate the ADS-B behavior over the $2.4 \mathrm{GHz}$ used by the radio chip.

- The collision detection can use information that the traffic sends by radio and the estimations of the state estimator to predict the future states of both MAVs. With a kinematic model, the detection could calculate some metrics to identify if a collision is imminent or not. 
- The low processing power of the quadcopters' main chip led to the search for avoidance algorithms that were not computationally costly. A hybrid approach of the geometric approach and the potential fields can be used.

- The new collision avoidance system should interface with the commander module to plan a collision-free path if a conflict is detected. That should guarantee that the MAVs involved will return to their initial trajectory after the system declares a situation clear of conflict, where imminent collision is no longer an issue. 


\section{Chapter 4}

\section{Implementation}

This chapter will expose all the implementation made within the scope of this work. Section 4.1 presents the implementation of ADS-B technology in the low-cost MAVs to enable their intercommunication. Section 4.2 shows the approach that MAVs use to detect collision scenarios. Finally, section 4.3 presents how, given a specific collision scenario, the avoidance maneuvers are derived from the Visual Flight Rules. It also explains how a hybrid approach to execute these maneuvers uses a mix of the Geometric-based and Artificial Potential Fields to evade the incoming traffic.

\subsection{ADS-B}

As exposed in the previous chapters, ADS-B can be used to send and receive relevant data (ID, position, velocity) to other aircraft that are equipped with the necessary systems on-board. The current hardware available in the MAV does not dispose of a chip that enables the communication at $1090 \mathrm{MHz}$ of the commercial ADS-B transceivers and transponders. So, for proof of concept, a mechanism that uses the MAV radio chip (nRF5182) was developed to simulate the same behavior as the ADS-B modules have, but on a frequency of $2.4 \mathrm{GHz}$.

This mechanism consists of the usage of a Peer-to-Peer (P2P) communication between the MAVs. A peer-to-peer network allows the MAVs to communicate without the need for a server. Unlike the client-server architecture used in the communication between the MAVs and the base station, there is no central server for processing requests in a P2P architecture. The MAVs directly interact with one another without the intervention of the base station [42]. A new task to handle this communication was implemented in the quadcopter firmware to receive and send broadcast messages in the environment automatically. These messages have the quadcopter ID, the current position (world reference from the indoor positioning system), and velocity in the world frame, both from the state estimator module, as requested by the ADS-B technology.

As the proposed mechanism uses the same radio chip used for the base station communication (CRTP Packets), the task that sends the data had to label these new P2P packets to be identified by the other MAVs as information coming from traffic nearby. As a result, the task that receives the packets had to identify these labeled messages and then store this data from other MAVs to be 


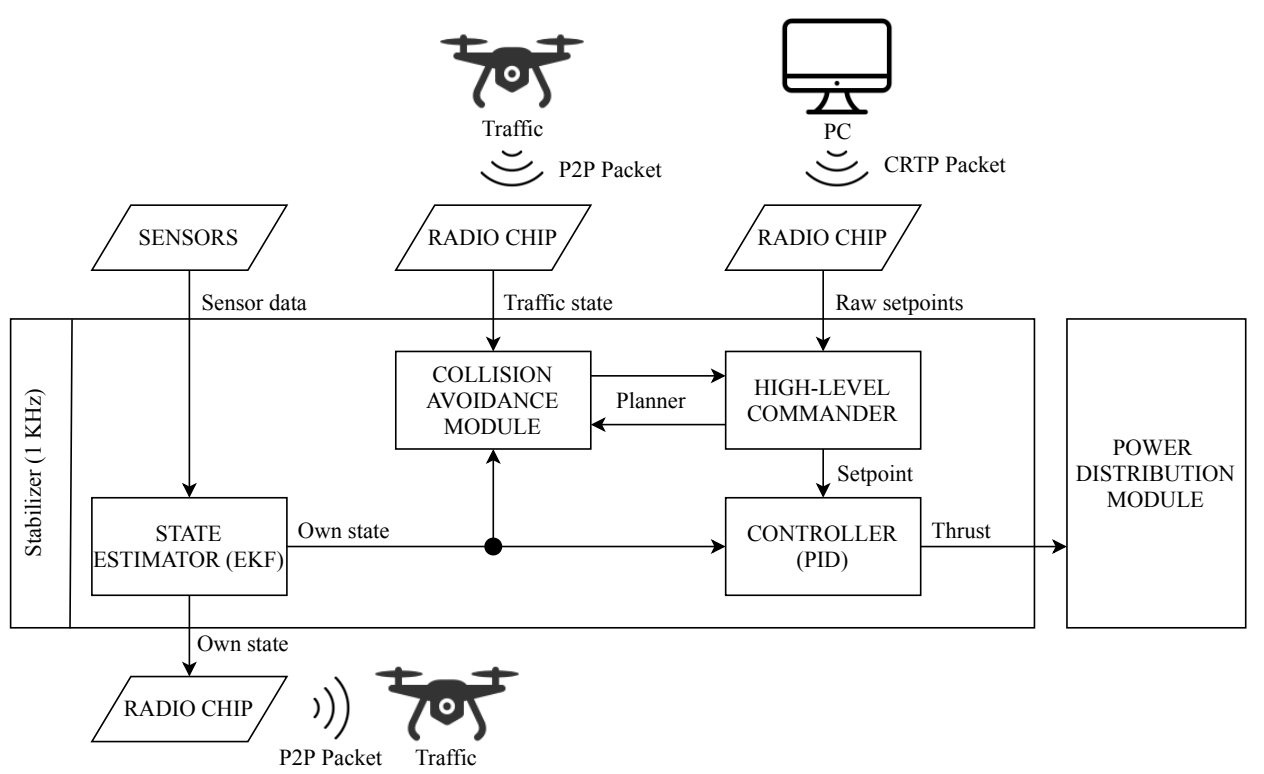

Figure 4.1: Overall system architecture operation

used by the detection algorithm. The states of each traffic quadcopter are stored inside a structure that keeps the most recent information about their current positions and velocities.

Figure 4.1 shows a diagram of the Stabilizer module of the quadcopters after the addition of the proposed new tasks that handle the collision avoidance system. The P2P module that adapts the ADS-B behavior interfaces with the system by receiving traffic states (id, position, and velocity) and storing them to be used by the new collision avoidance module. The other task of the P2P module is to take the quadcopter own states provided by the EKF, label them, and send in broadcast mode to the other MAVs in the vicinity. That enables a complete network of MAVs sharing their respective positions and velocities with all the others that are on its range.

\subsection{Conflict Detection}

The conflict detection of this work uses a geometric approach based on the flight plan sharing method explained at 2.2.4. The usage of the ADS-B provides the required data to enable a reliable projection of the future state of the traffic MAV with a kinematic model. Given that, this section presents a mathematical model derived from the kinematics of the MAVs that helps the system to predict a possible collision.

\subsubsection{Tait-Bryan angles convention}

For any derivation of a mathematical model of rigid bodies, a convention is needed for the different frames of the coordinate system since there are many possible configurations when dealing with problems in the three-dimensional space. One of the possible conventions is using the Tait-Bryan 


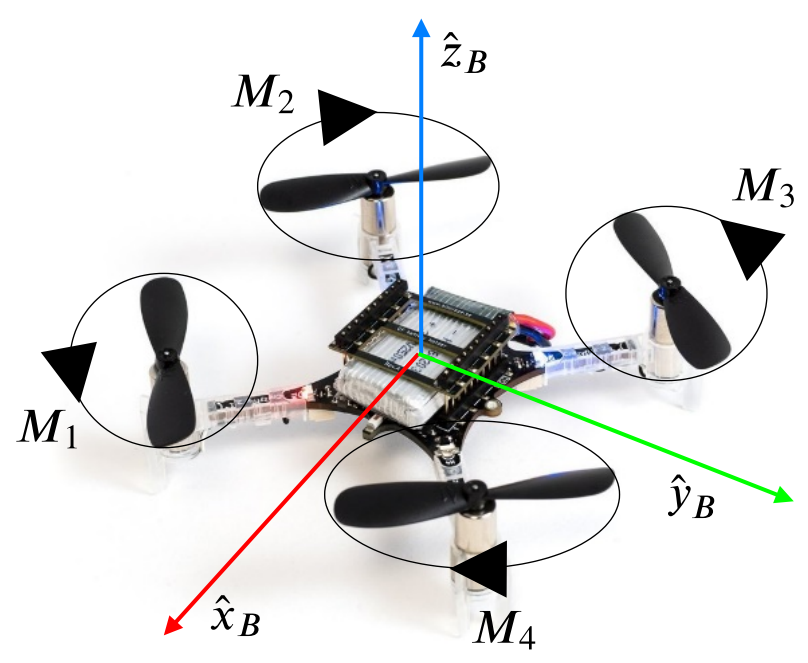

Figure 4.2: Quadcopter body coordinate system and motor configuration

angles, also known as Euler angles, widely used in aeronautics, which define the $\operatorname{roll}(\phi), \operatorname{pitch}(\theta)$, and yaw $(\psi)$ angles of the rigid body [30].

The position of the center of mass of the quadcopter within the global coordinate system will be denoted $\vec{p}[\mathrm{~m}]$. The center of the inertial coordinate system is placed in the quadcopter center of mass. The body frame represented in figure 4.2 is obtained with a rotation of the inertial coordinate system by the Tait-Bryan angles denoted $\vec{\eta}$ [rad].

$$
\vec{p}=\left[\begin{array}{l}
x \\
y \\
z
\end{array}\right], \quad \vec{\eta}=\left[\begin{array}{l}
\phi \\
\theta \\
\psi
\end{array}\right]
$$

The complete rotation from the inertial frame to the body frame is derived by applying three separate rotations. In this work, the ZYX rotation order was employed, referring to the sequence in which the rotations are applied. The complete rotational matrix $R_{G B}$ that indicates the rotation from the global to the body coordinates is given by

$$
R_{G B}=\left[\begin{array}{ccc}
c_{\theta} c_{\psi} & c_{\psi} s_{\phi} s_{\theta}-c_{\phi} s_{\psi} & c_{\phi} c_{\psi} s_{\theta}+s_{\phi} s_{\psi} \\
c_{\theta} s_{\psi} & c_{\phi} c_{\psi}+s_{\phi} s_{\theta} s_{\psi} & -c_{\psi} s_{\phi}+c_{\phi} s_{\theta} s_{\psi} \\
-s_{\theta} & c_{\theta} s_{\phi} & c_{\phi} c_{\theta}
\end{array}\right]
$$

Where $c_{i}=\cos (i)$ and $s_{i}=\sin (i)$ with $i$ corresponding to a specific angle $(\phi, \theta, \psi)$. 


\subsubsection{System Modeling}

Consider two UAVs with constant velocities toward their goal positions. The positions of UAV A and the traffic UAV B at a time $t \geq 0$, are given by

$$
\begin{aligned}
& \vec{s}_{a}(t)=\vec{p}_{a}+t \vec{v}_{a} \\
& \vec{s}_{b}(t)=\vec{p}_{b}+t \vec{v}_{b}
\end{aligned}
$$

Where $\vec{p}_{a}$ and $\vec{p}_{b}$ are the initial positions, and $\vec{v}_{a}$ and $\vec{v}_{b}$ are the velocity vectors of UAV A and UAV B in the global frame. To simplify the mathematical development, this work considers the use of a relative coordinate system where UAV A is static at the center of this system (body frame explained at point 4.2.1). In this relative system, UAV B is located at $\vec{s}=\vec{p}_{b}-\vec{p}_{a}$ and it moves at a relative velocity $\vec{v}=\vec{v}_{b}-\vec{v}_{a}$. These relations define the relative position of the traffic UAV B with respect to UAV A at any time $t$ as:

$$
\vec{s}(t)=\vec{s}+t \vec{v}
$$

The range $r(t)$ to the traffic is calculated simply by finding the norm of $\vec{s}(t)$.

$$
r(t)=\|\vec{s}(t)\|=\sqrt{\|\vec{s}\|^{2}+2 t \vec{s} \cdot \vec{v}+t^{2}\|\vec{v}\|^{2}}
$$

The derivative of $r(t)$ with respect to $t$ gives the rate that the UAVs are approaching or distancing each other.

$$
\dot{r}(t)=\frac{\vec{s} \cdot \vec{v}+t\|\vec{v}\|^{2}}{\|\vec{s}(t)\|}
$$

\subsubsection{Time to the Closest Point of Approach}

The time $\tau(\vec{s}, \vec{v})$ at the Closest Point of Approach (CPA), when $r(t)$ reaches its minimum, is the time $t$ that satisfies $\dot{r}(t)=0$. Therefore,

$$
\tau(\vec{s}, \vec{v})=-\frac{\vec{s} \cdot \vec{v}}{\|\vec{v}\|^{2}}
$$

In equation 4.7, the dot product of vectors $\vec{s}$ and $\vec{v}$ is what defines if $\tau(\vec{s}, \vec{v})$ is positive, negative, or zero ${ }^{1}$. By taking the definition of the dot product between two vectors $\vec{s} \cdot \vec{v}=\|\vec{s}\|\|\vec{v}\| \cos (\beta)$, where $\beta$ is the angle between these vectors, it can be implied that,

$$
\left\{\begin{array}{lll}
\tau(\vec{s}, \vec{v})>0 & \text { if } & \cos (\beta)<0 \\
\tau(\vec{s}, \vec{v})<0 & \text { if } & \cos (\beta)>0 \\
\tau(\vec{s}, \vec{v})=0 & \text { if } & \cos (\beta)=0
\end{array}\right.
$$

\footnotetext{
[43].

${ }^{1}$ By convention, $\tau(\vec{s}, \vec{v})$ is also defined 0 when the velocities of both UAV are identical, i.e., $\tau(\vec{s}, \vec{v})=0$, if $\|\vec{v}\|^{2}=0$
} 


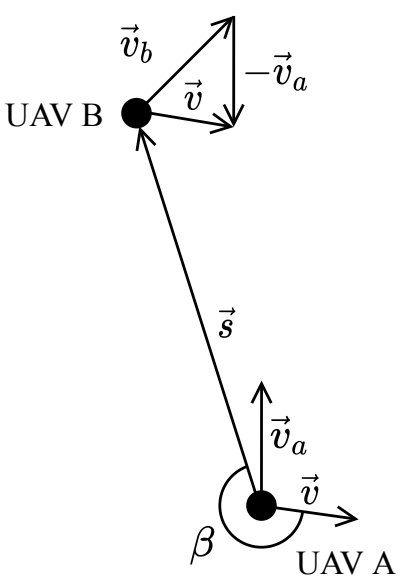

a)

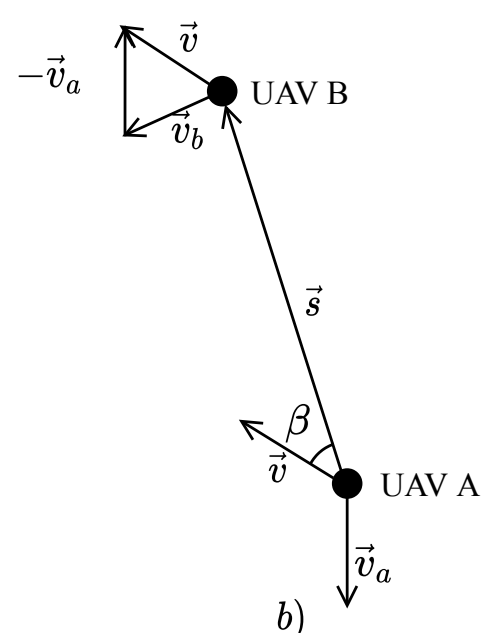

b)

Figure 4.3: System modeling to determine $\tau(\vec{s}, \vec{v})$ (a) UAVs getting closer, (b) UAVs moving away

Figure 4.3 shows two scenarios where the drones are approaching or distancing each other. It is easy to visualise that when the UAVs are getting closer, $\cos (\beta)$ is negative, i.e. $\tau(\vec{s}, \vec{v})>0$, and when the UAVs are moving away, $\cos (\beta)$ is positive, i.e. $\tau(\vec{s}, \vec{v})<0$. Therefore, when $\tau(\vec{s}, \vec{v}) \geq 0$, it is necessary to check if there is a chance to have a collision or not. When $\tau(\vec{s}, \vec{v})<0$, there is no risk to have an event of a conflict, and the UAVs can continue their normal trajectories.

\subsubsection{Safe zone}

When the traffic is approaching, these UAVs are considered in a possible collision scenario if they are inside each other's safe zone. The volume of the safe zone is usually dependent on the altitude, speed, and heading of the aircraft involved in the encounter. For simplicity, the safe zone in this works is a sphere in which radius that varies according to the current velocity of the UAV.

$$
r_{\text {safe }}=\left\|\vec{v}_{G}\right\| t_{d}
$$

Where $r_{\text {safe }}$ is the radius of the safe zone, $\left\|\vec{v}_{G}\right\|$ is the magnitude of its velocity vector in the global frame, and $t_{d}$ is the detection time. Typically the detection time is fixed to be around 20-30 seconds to enable big commercial aircraft to perform their maneuvers safely [44]. Given the small size and low inertia of the MAVs used in this work, $t_{d}$ was fixed to be 3 seconds for compliance with the test environment.

\subsubsection{Separation distance}

When the two UAVs are approaching and inside each other safe zone, if we calculate the separation distance between them in the so-called CPA, where this distance will be minimal, it is possible to 


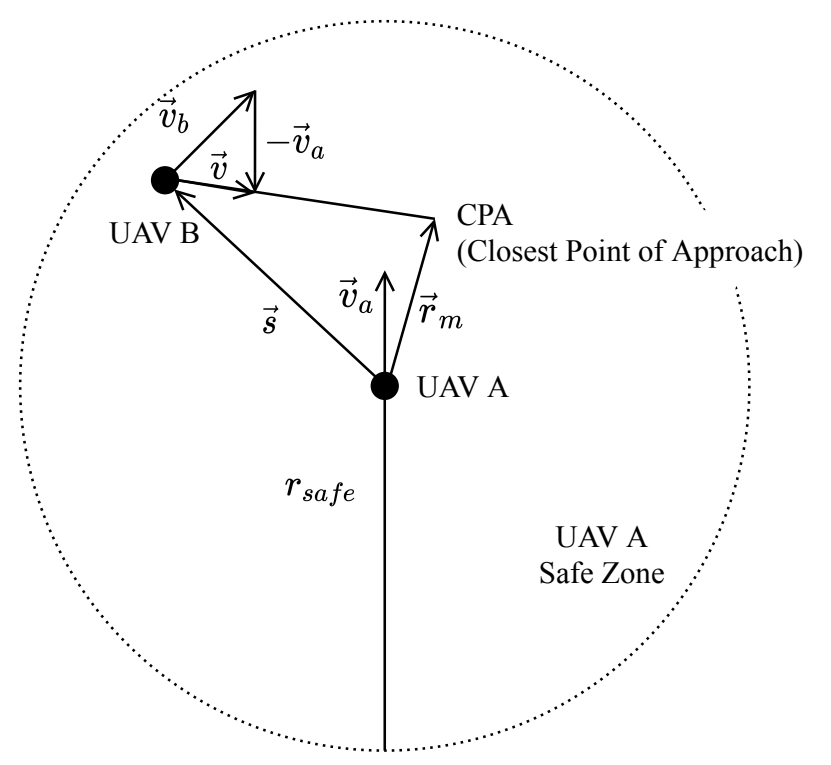

Figure 4.4: System modeling to determine $r_{s a f e}$ and $\vec{r}_{m}$

judge whether a collision will occur or not. The miss-distance vector $\vec{r}_{m}$ is defined as:

$$
\vec{r}_{m}=\vec{s}+\vec{v} \cdot \tau(\vec{s}, \vec{v})
$$

If the magnitude of $\vec{r}_{m}$ is less than a specified threshold, $r_{\text {min }}$, that tells the minimum separation distance, the two UAVs are considered in a conflict condition. Given the small size of the MAVs used in this work, a threshold that is the double of the diameter of the Crazyflie, i.e., $r_{\min }=0.3[\mathrm{~m}]$, was considered. Therefore, for the conflict condition:

Collision scenario $=\left\{\begin{array}{ll}\text { true } & \text { if } \\ \text { false } & \text { otherwise }\end{array}(\vec{s}, \vec{v})>0\right.$ and $r(t)<r_{\text {safe }}$ and $\left\|\vec{r}_{m}\right\|<r_{\text {min }}$

This condition detects if the collision avoidance module may intervene in the current setpoint, planning another trajectory that will take the UAV thought a safe path. This planning consists of the use of the Visual Flight Rules for collision scenarios.

\subsection{Collision Avoidance Maneuver}

The implementation of the avoidance maneuvers consists of two approaches that together enable an appropriate and safe motion of the UAVs involved in the collision. An extension of the geometric approach of the collision detection is used to calculate an evasive waypoint to resolve the conflict and prevent the collision while adhering to the Visual Flight Rules of ICAO Rules of the Air. This approach is fused with an Artificial Potential Field (APF) method that controls the velocity and makes the avoidance maneuver smoother. 


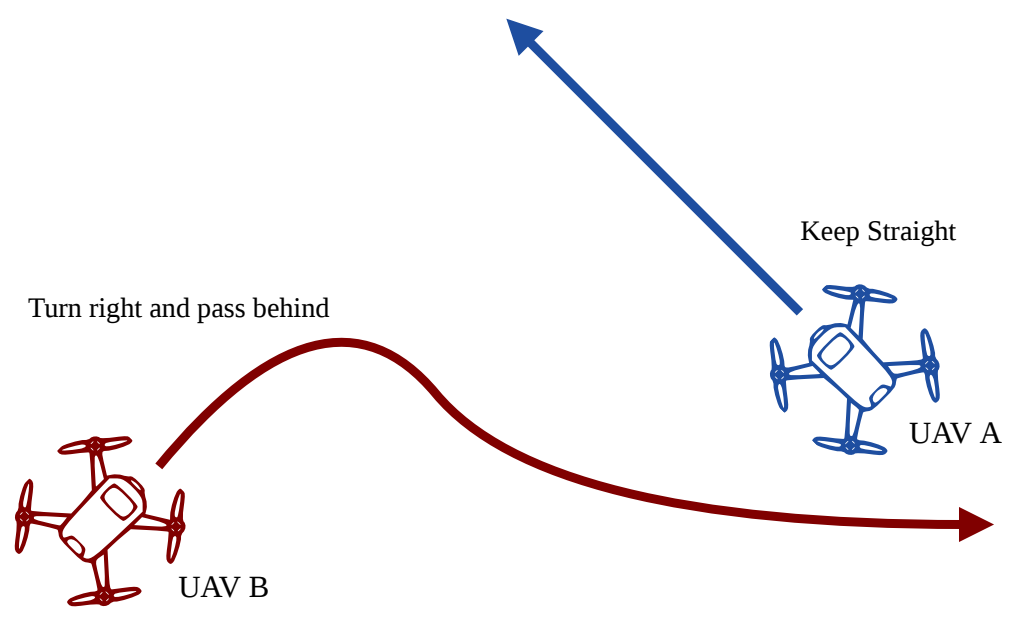

Figure 4.5: Collision avoidance in a converging traffic scenario

\subsubsection{Visual Flight Rules}

This work investigates the VFR of ICAO Rules of the Air standard that specifies two general situations for collisions between aircraft: Converging traffic and Head-on approach [11]. For compliance with the Rules of the Air convention, the traffic relative to the UAV must be identified before an appropriate evasive maneuver can be planned and performed. Therefore, the criteria for each of the two scenarios considered must be defined and validated to determine the current traffic state for each UAV.

\subsubsection{Converging traffic}

The Rules of the Air standard defines that "When two aircraft are converging at approximately the same level, the aircraft that has the other on its right shall give way" [11]. This implies that the UAV that is on the right has the "right-of-way" and shall maintain its heading and speed, as the other starts the evasion maneuver. Thus, to determine if traffic is converging from left or right, the relative bearing to the traffic is calculated:

$$
\theta=\arctan \left(\frac{s_{y}}{s_{x}}\right)
$$

Where $\theta$ is the angle to the traffic and $s_{x}, s_{y}$ are the $\mathrm{x}$ and $\mathrm{y}$ components of the relative position vector $\vec{s}(t)$. If the angle to the traffic is between $0^{\circ}$ to $180^{\circ}$ then the approaching traffic is on the right. Alternatively, if the relative bearing is from $0^{\circ}$ to $-180^{\circ}$ the traffic is on the left. The clockwise convention is used following the compass bearings that are measured clockwise.

In figure 4.5, UAV B has traffic on its right, so it should change its trajectory to the right and pass behind UAV A. On the other hand, UAV A has traffic on its left and therefore is not required to maneuver away from UAV B as exposed in the Rules of the Air standard. 


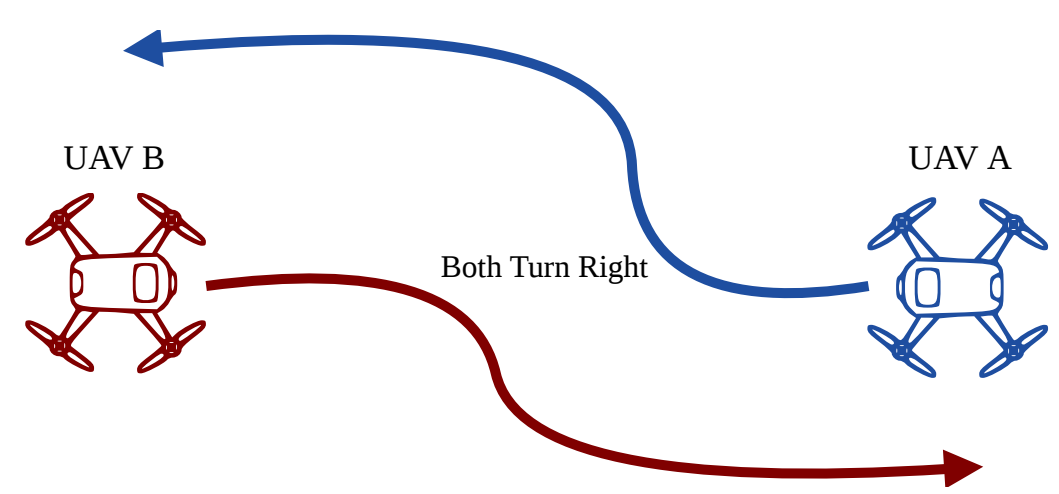

Figure 4.6: Collision avoidance in a head-on traffic scenario

\subsubsection{Head-on traffic}

For UAVs that are on a frontal collision course, both must maneuver to the right to avoid the conflicting traffic. Thus, we have to determine if the traffic is in this situation or not. First, we take the heading of each UAV by:

$$
\alpha=\arctan \left(\frac{v_{y}}{v_{x}}\right)
$$

Where $\alpha$ is the heading angle and $v_{x}, v_{y}$ are the $\mathrm{x}$ and $\mathrm{y}$ components of the UAVs velocity vector in the global frame $\vec{v}_{G}$. Considering a scenario with two UAVs, one must compare its reciprocal heading, i.e. $\alpha_{s}+/-180^{\circ}$, with the inbound traffic heading $\alpha_{t}$.

$$
\text { Head-on scenario }=\left\{\begin{array}{ll}
\text { true } & \text { if } \\
\text { false } & \text { otherwise }
\end{array}\left|\alpha_{t}-\alpha_{s} \pm 180^{\circ}\right|<15^{\circ}\right.
$$

All the traffic UAVs that have a heading angle within $+/-15^{\circ}$ of the reciprocal heading are considered as head-on traffic. The value of $+/-15^{\circ}$ was chosen after a sensitivity study that found that this value is the optimal detection envelope for the head-on traffic scenario [44].

Figure 4.6 shows a scenario where both UAVs are opposite each other on a head-on collision course. In this situation, is the responsibility of both UAVs to turn to the right and avoid the collision.

\subsubsection{Evasive waypoint approach}

Given the presence of frontal or right-hand traffic, it is necessary to calculate an evasive waypoint so that there is no collision between the UAVs. This approach involves considering the geometric scenario defined in section 4.2 , therefore being an extension of it.

To specify the new directions in which the UAVs avoid the collision, the evasive waypoint is calculated to be perpendicular to the Line of Sight (LOS) [44]. Given a specific time $t$, when the 


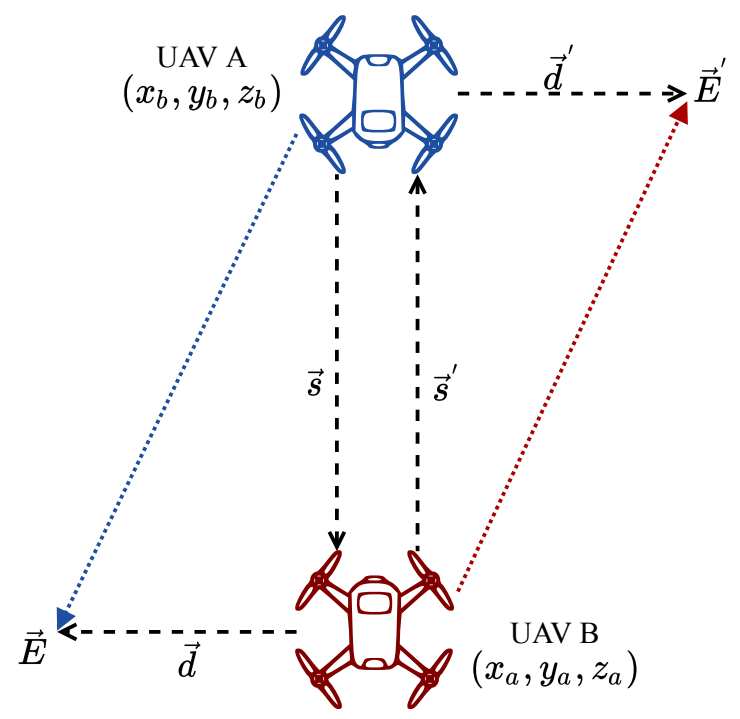

Figure 4.7: Scenario for the evasive waypoint $\vec{E}$ determination

system detects a possible collision between the quadcopters, the distance to the traffic is calculated using the position vectors of both UAVs:

$$
\vec{s}=\vec{s}_{b}-\vec{s}_{a}
$$

Where $\vec{s}_{b}$ is the position vector of the traffic UAV B, $\vec{s}_{a}$ is the position vector of UAV A and $\vec{s}$ is the calculated vector from UAV A to traffic. The evasive waypoint $\vec{E}$ is on the right-hand side of the traffic and perpendicular to LOS $\vec{s}$. The direction unit vector $\hat{d}$ that defines this condition is calculated by:

$$
\hat{d}=\hat{u} \times-\hat{s}
$$

Where $\hat{s}$ is a unit vector in the direction of $\vec{s}$ and $\hat{u}=\left[\begin{array}{lll}0 & 0 & 1\end{array}\right]^{T}$ is the unit vector in the $z$ direction (upwards). From this direction, vector $\vec{d}$ to the evasive waypoint $\vec{E}$ can be calculated as:

$$
\vec{d}=E_{d} \hat{d}
$$

Where $E_{d}$ is a constant to define how far the evasive waypoint should be from the traffic. This constant is fixed to be $E_{d}=0.8[\mathrm{~m}]$ to guarantee the minimum separation distance of $r_{\min }=0.3[\mathrm{~m}]$. Therefore, evasive waypoint $\vec{E}$ is:

$$
\vec{E}=\vec{s}_{b}+\vec{d}
$$

\subsubsection{Potential field approach}

After some initial tests with the algorithm that calculates the evasion points covered in 4.3.2, there was a need to incorporate a method to control the MAV speed when performing the collision avoid- 
ance maneuver. Upon receiving the new evasive waypoint, the MAV started to move too roughly, and this behavior provided the system with a certain degree of insecurity in evasive motion. For this reason, a way was sought to smooth the movement of the evasive maneuver using the artificial potential field method.

Let $\vec{p}$ represent the position of the MAV, considered as a particle moving in the 3-dimensional space $\mathbb{R}^{3}$. For simplicity of the mathematical development, let's consider that the MAV and its intruders are at the same height and both are moving in a plane, i.e. $\mathbb{R}^{2}$, and that the quadcopter's pose is defined by the tuple $\vec{p}=(x, y)$. The total potential field $U(\vec{p})$, which each MAV is modeled as a moving particle under the influence of, is a combination of the individual potential field:

$$
U(\vec{p})=U_{a t t}(\vec{p})+\sum_{i} U_{r e p}(\vec{p})
$$

Where $U_{a t t}(\vec{p})$ is the attractive potential that drives the MAV to the desired waypoint and $U_{\text {rep }}(\vec{p})$ is the repulsive potential generated by the intruder $i$.

If $U(\vec{p})$ is differentiable, at each position $\vec{p}$, the gradient of the potential filed, denoted by $\nabla U(\vec{p})$, is a vector that points in the direction that locally increases $U(\vec{p})$. In robotics, it's common to consider the attractive potential as zero at the goal and to increase as the robot is far away from it. The inverse happens with the repulsive potential as it is very high (infinity) in the close vicinity of the obstacles and decreases as the distance to them increases [45]. Therefore, the virtual force that drives the MAV to the waypoint is the negative gradient of the artificial potential:

$$
\vec{F}(\vec{p})=\vec{F}_{\text {att }}(\vec{p})+\vec{F}_{\text {rep }}(\vec{p})=-\nabla U_{\text {att }}(\vec{p})-\nabla U_{\text {rep }}(\vec{p})
$$

The force $\vec{F}(\vec{p})$ is a vector that points in the direction that, at each $\vec{p}$, locally maximally decreases $U(\vec{p})$. This force can be considered as the velocity vector that drives the MAV to its goal.

\subsubsection{Attractive potential field}

The attractive potential field is responsible for moving the MAV to the desired evasive waypoint. There are several fields of attraction that can be chosen, the most usual being the parabolic, which grows in proportion to the square of the distance to the waypoint.

$$
U_{\text {att }}(\vec{p})=\frac{1}{2} k_{\text {att }} d_{\text {goal }}^{2}(\vec{p})
$$

Where $k_{\text {att }}>0$ is a scaling constant and $d_{\text {goal }}(\vec{p})=\left\|\vec{p}-\vec{p}_{\text {goal }}\right\|$ is the Euclidean distance of the current position of the MAV to the goal. Therefore, the gradient of $U_{\text {att }}(\vec{p})$ :

$$
\nabla U_{\text {att }}(\vec{p})=k_{\text {att }}\left(\vec{p}-\vec{p}_{\text {goal }}\right)
$$

The attractive force is the negative gradient of the attractive potential, then:

$$
\vec{F}_{\text {att }}(\vec{p})=-\nabla U_{\text {att }}(\vec{p})=k_{\text {att }}\left(\vec{p}_{\text {goal }}-\vec{p}\right)
$$




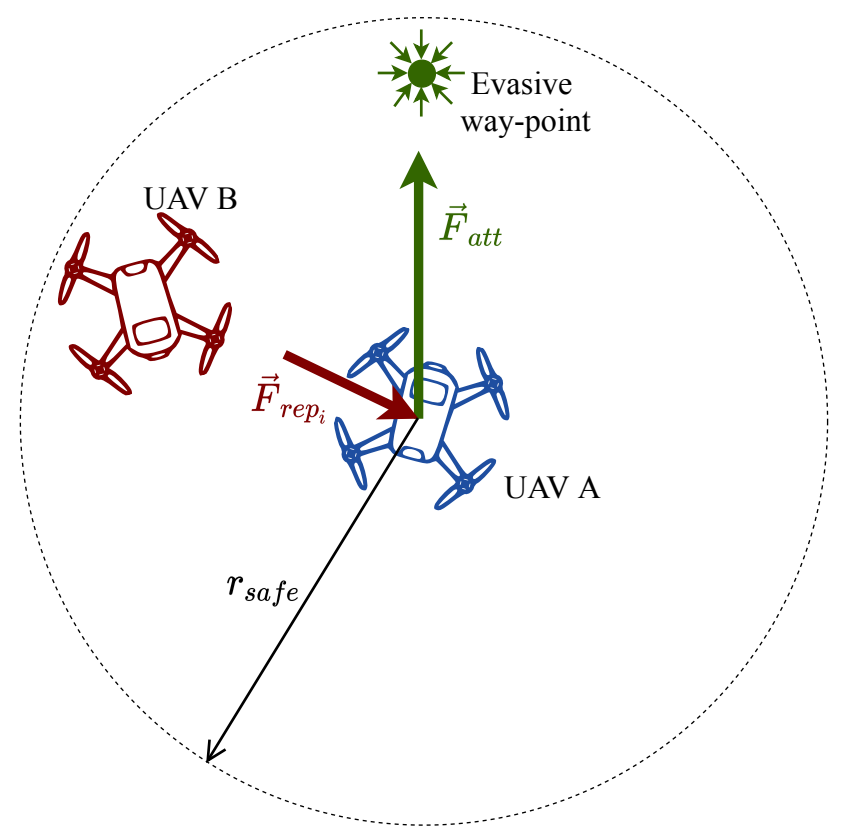

Figure 4.8: Artificial potential field vector diagram

\subsubsection{Repulsive potential field}

The repulsive potential has the premise of keeping the quadcopter away from other MAVs around it. The influence of this potential field grows as the distance between the quadcopter and the intruder decreases. Given the linear nature of the problem, the repulsive potential is the sum of the repellent effects of all MAVs that are in the quadcopter's vicinity:

$$
U_{\text {rep }}(\vec{p})=\sum_{i} U_{\text {rep }}(\vec{p})
$$

It is reasonable to consider that the influence of an intruder's repulsive potential is limited to a determined minimum distance $r_{\text {safe }}$. If an intruder is too far away, there is no reason for it to repel the quadcopter. To account for this space bounded influence, a possible repulsive potential generated by intruder $i$ is:

$$
U_{\text {repi }}(\vec{p})=\left\{\begin{array}{lcc}
\frac{1}{2} k_{\text {rep }}\left(\frac{1}{d_{\text {int }}(\vec{p})}-\frac{1}{r_{\text {safe }}}\right)^{2} & \text { if } & d_{\text {int }}(\vec{p})<r_{\text {safe }} \\
0 & \text { otherwise }
\end{array}\right.
$$

Where $d_{\text {int }}(\vec{p})=\left\|\vec{p}-\vec{p}_{\text {int }}\right\|$ is the Euclidean distance to the intruder $i, k_{\text {rep }}$ is a scaling gain and $r_{\text {safe }}$ is the intruder influence threshold. The repulsive force is the negative gradient of the repulsive potential, i.e. $\vec{F}_{r e p_{i}}(\vec{p})=-\nabla U_{\text {rep }}(\vec{p})$, therefore:

$$
\vec{F}_{\text {rep }}(\vec{p})=\left\{\begin{array}{lc}
k_{\text {rep }}\left(\frac{1}{d_{\text {int }}(\vec{p})}-\frac{1}{r_{\text {safe }}}\right) \frac{\vec{p}-\vec{p}_{\text {int }}}{d_{\text {inti }}^{3}(\vec{p})} \quad \text { if } & d_{\text {int }}(\vec{p})<r_{\text {safe }} \\
0 & \text { otherwise }
\end{array}\right.
$$




\subsubsection{Double integrator model}

Usually, the virtual force $\vec{F}(\vec{p})$ is set to be the velocity vector that drives the MAV to the goal with a magnitude that decreases when the MAV approaches the goal. Unfortunately, the commander module of the Crazyflie only accepts position commands to be injected into the PID controllers explained at 3.1.2.4. This limitation forced that the values obtained for $\vec{F}(\vec{p})$ have to be integrated over time to obtain intermediate position setpoints to the goal.

The approach to the problem was to represent each quadcopter mathematically by the double integrator model [46]

$$
\begin{gathered}
\dot{p}_{i}=v_{i} \\
\dot{v}_{i}=\frac{1}{M_{i}} u_{i}
\end{gathered}
$$

Where $p_{i} \in \mathbb{R}^{3}$ is the position, $v_{i} \in \mathbb{R}^{3}$ is the velocity, $M_{i}$ is the mass, and $u_{i} \in \mathbb{R}^{3}$ is the virtual force (control input) that drives the $i^{\text {th }}$ quadcopter to its goal. The equation above implies that $u_{i}=M_{i} \ddot{p}$ (force is mass times acceleration). To get the intermediate positions to get to the goal, the acceleration must be integrated twice, therefore:

$$
\begin{gathered}
\vec{p}_{\text {imd }}=\vec{p}+\vec{v}_{\text {des }} \Delta t \\
\vec{v}_{d e s}=\vec{v}+\frac{\vec{F}(\vec{p})}{M_{i}} \Delta t
\end{gathered}
$$

Where $\vec{p}_{i m d}$ is the intermediate position setpoint, $\vec{p}$ and $\vec{v}$ are the current position and velocity of the MAV, $\vec{v}_{\text {des }}$ is the desired velocity, $\vec{F}(\vec{p})=\vec{F}_{\text {att }}(\vec{p})+\vec{F}_{\text {rep }}(\vec{p})$ is the virtual force, $M_{i}=0.03[\mathrm{~kg}]$, and $\Delta t=t_{k}-t_{k-1} \approx 1[\mathrm{~ms}]$ is the discrete time integration interval. 


\section{Chapter 5}

\section{Experiments and results}

In this chapter, we present all the experiments conducted to validate the implemented methods and algorithms exposed in the last chapter. In section 5.1 the experiments that used the flight simulator are presented. Section 5.2 provides the real-world experiments conducted in the tests arena located at Altran's office. Finally, section 5.3 presents a brief overview of the results obtained.

\subsection{Flight simulator experiments}

To provide a first test environment for the methods and algorithms exposed in the last chapter, the software-in-the-loop (SITL) simulator stated in 3.2.3 was used. All the real-world experiments that put both MAVs into collision scenarios were also firstly tested in this simulation environment. As both results lead to the same conclusions, this section only provides the tests that have not been done in the part of the real-world experiments.

\subsubsection{Artificial Potential Fields}

In the last chapter, the double integrator model was used to solve the problem of interface between the APF approach and the position commands used by the HLC module. However, tests with the proposed solution were needed to validate the overall behavior that integrated the acceleration twice to obtain the intermediate positions to the goal.

These experiments consisted only of moving the simulated quadcopter thought the environment trying to observe how was the evolution over time of the MAV in the three different axes of motion. The base station provided position setpoints over distinct moments. That enables seeing the behavior of the quadcopter transient and steady-state responses. The MAV had on its vicinity another quadcopter that was considered as traffic to be avoided. That is intended to test both the attractive and repulsive potentials. Given the small size of the quadcopters, the influence of the intruder's repulsive potential was determined by $r_{s a f e}=0.4$ minimum distance. In a first analysis, the attractive and repulsive gains were set to $k_{\text {att }}=5$ and $k_{\text {rep }}=0.1$ by intuition.

Initial tests showed a behavior with an inadequate steady-state response. Figure 5.1 represents the position evolution of one MAV on the Gazebo simulator. For $t>8 \mathrm{~s}$, when the APF algorithm 

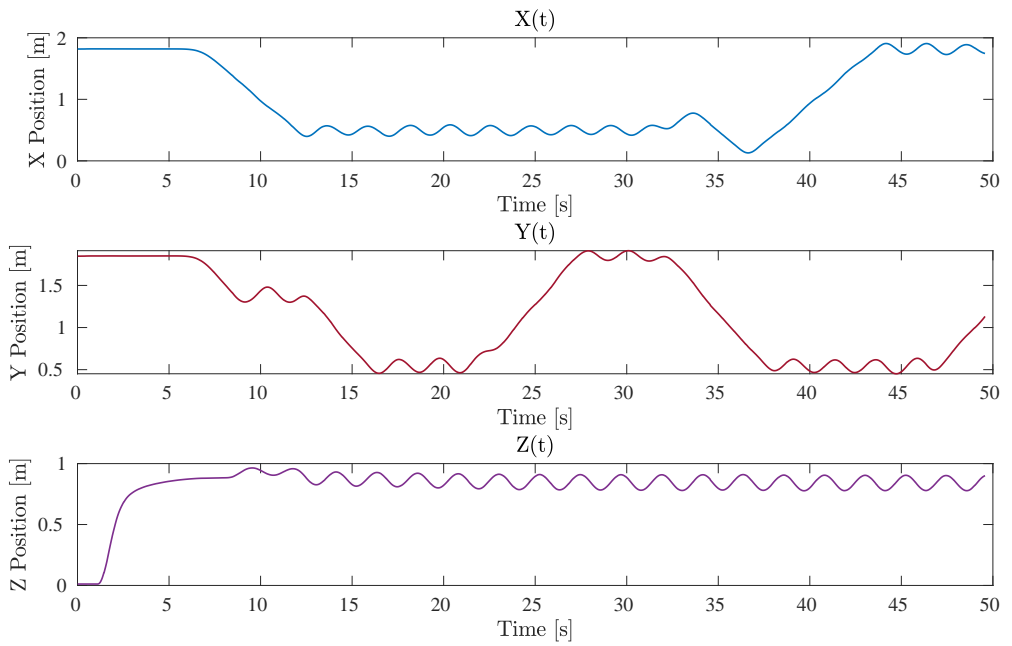

Figure 5.1: Position vs. time of the MAV without velocity error term

was enabled, the quadcopter was with an oscillatory motion that could generate an unsafe avoidance maneuver. The same behavior occurred even when the values for the attractive gain $k_{\text {att }}$ were set to different values. The approach to reducing that behavior was to add a term to include the error of the desired velocity on equation 4.20.

$$
\vec{F}_{\text {att }}(\vec{p})=k_{\text {att }}\left(\vec{p}_{\text {goal }}-\vec{p}\right)+k_{d}\left(\vec{v}_{\text {des }}-\vec{v}\right)
$$

Where $k_{d}$ is a scaling gain, $\vec{v}$ is the current velocity of the quadcopter, and $\vec{v}_{d e s}$ is the desired velocity calculated with equation 4.26 from the previous loop interaction. A new experiment, with the same conditions as the first, was conducted. The scaling gain $k_{d}$ was set to be half of the attractive gain $k_{\text {att }}$.

Figure 5.2 shows the motion of the quadcopter in the Gazebo simulator with the velocity error term addition. The results showed that the motion was a lot more stable, without oscillations on any of the axes. Fine-tuning was required to obtain these results, and the final values of the scaling gains are presented in table 5.1.

Table 5.1: Final values of scaling gains after fine-tuning

\begin{tabular}{cc}
\hline Scaling Gain & Value \\
\hline$k_{a t t}$ & 8 \\
$k_{d}$ & 4 \\
$k_{\text {rep }}$ & 0.1 \\
\hline
\end{tabular}

Finally, to enable a controlled speed over the quadcopter motion, some limits were set for the max and min values of $\vec{F}(\vec{p})$, i.e $+/-0,03[\mathrm{~N}]$, and $\vec{v}_{d e s}$, i.e $+/-0,25[\mathrm{~m} / \mathrm{s}]$. These approaches successfully solved the problems of abrupt motion and big velocities that drove the MAVs for unsafe collision avoidance maneuvers. 

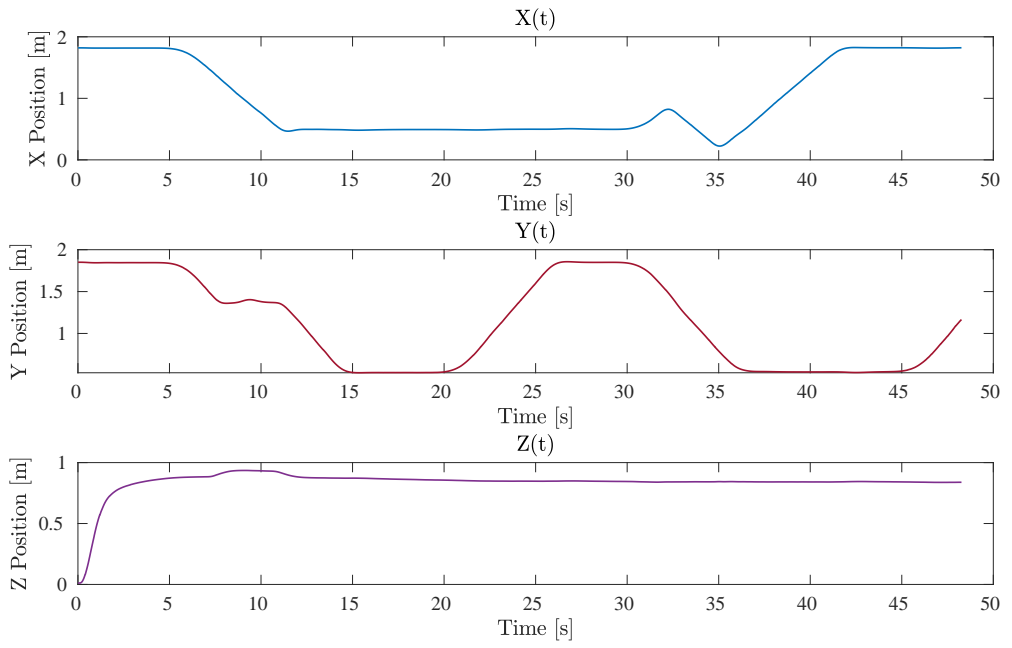

Figure 5.2: Position vs. time of the MAV with the velocity error term

\subsection{Real-world experiments}

This section is intended to present the real-world experiments conducted in the scope of this dissertation. First, it will be presented the results from the adaptation of the ADS-B technology for the micro quadcopters and the choices that were made to interfere as little as possible with its operation.

The flight experiments will also be presented to show the detection and avoidance strategies adopted. These were tested for three scenarios that are supposed to show the robustness of the solutions, namely head-on, converging traffic, and close approach scenarios. The experiments were set at Altran's drone arena. That is an indoor $2.2 \mathrm{~m}$ x $2.4 \mathrm{~m} \times 2.3 \mathrm{~m}$ test arena where the MAVs have all the freedom to navigate and perform the avoidance maneuvers. It is relevant to mention that the test arena provided a stable flight for the MAVs. That was essential for the successful collision avoidance experiments as a stable flight provided the focus of the work to be into the relevant parts.

\subsubsection{Peer-to-peer communication}

Some experiments were conducted to test the Peer-to-peer (P2P) communication implemented in the quadcopters to simulate the same behavior of the ADS-B technology. As the same radio chip that communicates with the base station, exposed on point 3.2.2, is the one that sends/receives the new P2P packets, the experiments tried to verify if there was strong interference between them.

Table 5.2 shows the average rate of received P2P packets between two MAVs for different distances and with the presence or not of computer radio communication ${ }^{1}$. In these experiments,

\footnotetext{
${ }^{1}$ To retrieve data without the computer radio communication, the MAVs were connected with the base station by USB.
} 
the quadcopters were in the ground distancing each other from $0.3 \mathrm{~m}$ to $1.5 \mathrm{~m}$. The arrival of packets was watched for a time of 60 seconds while the MAVs sent messages at a rate of $10 \mathrm{~Hz}$.

Table 5.2: Average rate of received $\mathrm{P} 2 \mathrm{P}$ packets with the same sending frequency

\begin{tabular}{lccc}
\hline $\begin{array}{l}\text { Communication } \\
\text { with computer }\end{array}$ & $300 \mathrm{~mm}$ & $750 \mathrm{~mm}$ & $1500 \mathrm{~mm}$ \\
\hline Enable & $63.29 \%$ & $29.61 \%$ & $0.00 \%$ \\
Disable & $100.0 \%$ & $98.91 \%$ & $87.33 \%$ \\
\hline
\end{tabular}

As expected, the presence of the base station communication is harmful to the interaction between the two MAVs. That can be explained as the P2P packets are sent and received on the same channel as the currently configured radio link between PC (base station) and quadcopter. That resulted in an enormous loss of P2P packets, as the computer is always querying the MAVs to get their current flight status, keeping the channel busy.

A new experiment was conducted to reduce the impact of that on the overall system behavior. The time that the computer waits to retrieve new data from the MAVs was increased to a fixed interval of $0.2 \mathrm{~s}$, and the frequencies that each quadcopter send P2P messages were changed $(15.15 \mathrm{~Hz}$ and $12.98 \mathrm{~Hz})$ to reduce collisions of packets. Table 5.3 shows the rates of received P2P packets in that new configuration.

Table 5.3: Average rate of received P2P packets with different sending frequency

\begin{tabular}{llll}
\hline Communication & \multicolumn{3}{c}{ Distance } \\
with computer & $300 \mathrm{~mm}$ & $750 \mathrm{~mm}$ & $1500 \mathrm{~mm}$ \\
\hline Enable & $85.72 \%$ & $78.94 \%$ & $62.50 \%$ \\
Disable & $100.0 \%$ & $100.0 \%$ & $96.21 \%$ \\
\hline
\end{tabular}

Although the results were improved, the $62.5 \%$ rate is still far from the acceptable rate that provides reliable data for real-time collision avoidance algorithms. However, the only way to retrieve data from the quadcopters for the flight experiments is by using that base station communication. Moreover, this limitation can be classified as a specific issue of the MAVs used in this work. In a real scenario, the channel used for ADS-B communication is different from the one used to communicate with the base station. Given that, aiming to interfere as little as possible in the ADS-B operation, but with the need to get data for the results, the retrieval of flight status was maintained with a minimum interval of $0.2 \mathrm{~s}$, providing enough data for the collision avoidance experiments.

\subsubsection{Head-on collision}

The conducted experiment used two quadcopters referenced as MAV A and MAV B. Firstly, the computer commands that both take-off and send each one to its respective initial position $\vec{p}_{a}=$ $(0.50,0.55,0.84)$ for MAV A and $\vec{p}_{b}=(1.82,1.85,0.84)$ for MAV B. 
Next, the base station commands that the quadcopters have to exchange their positions, i.e $\vec{p}_{\text {goal }_{a}}=\vec{p}_{b}$, and $\vec{p}_{\text {goal }_{b}}=\vec{p}_{a}$. As the MAVs will try to move to the goal on a straight line, it will result in a collision on half of the way to the setpoint.

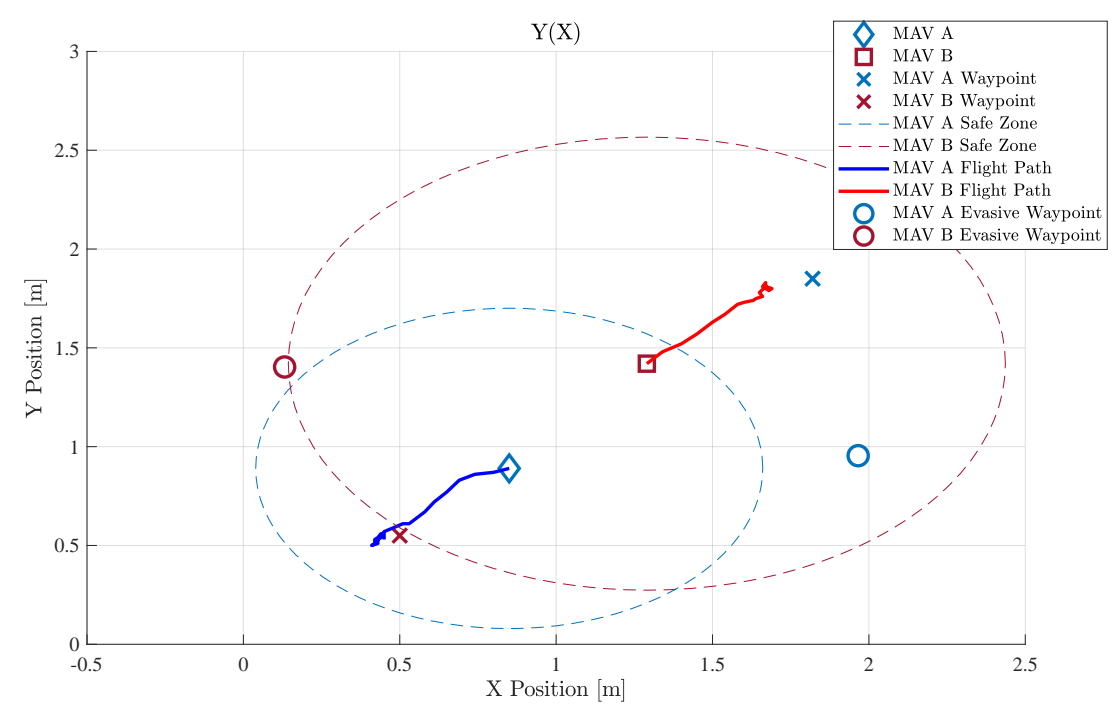

Figure 5.3: Initial detection and beginning of evasive maneuver of the head-on collision

Figure 5.3 shows that both MAVs detect each other since they are on a collision course and the intruders are inside their respective Safe Zones. An appropriate evasive waypoint is created for each quadcopter to avoid the collision.

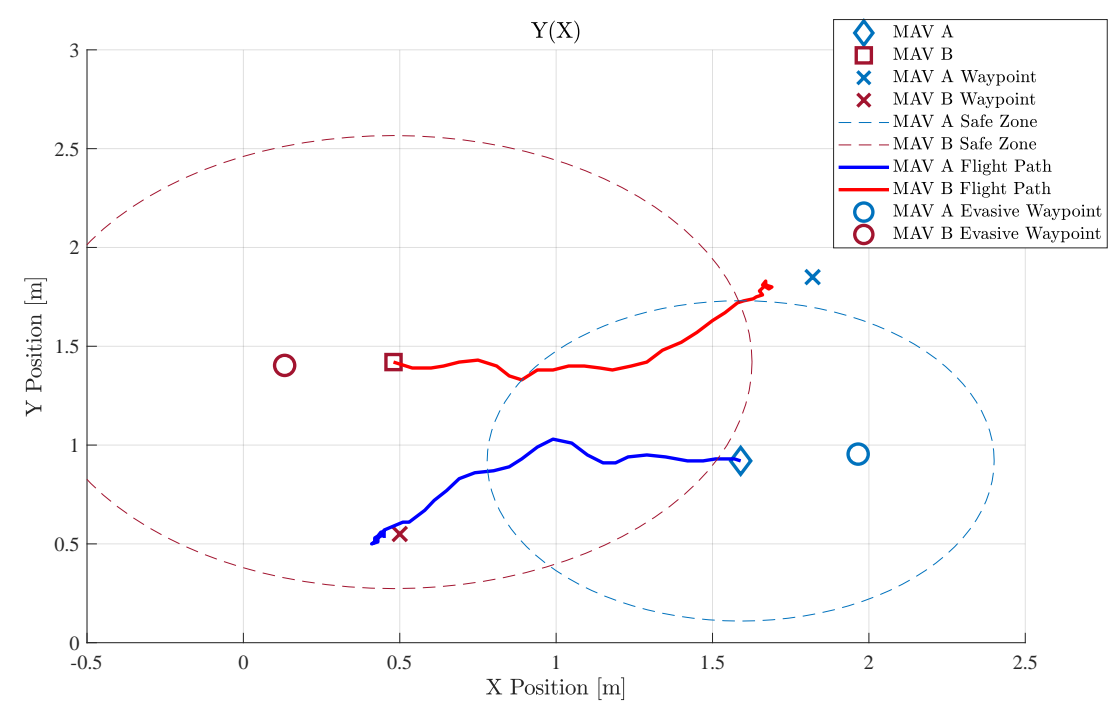

Figure 5.4: Evasive maneuver of the head-on collision scenario

Figure 5.4 represents the moment when both MAVs are following their respective evasive waypoints and preventing a collision between them. At this moment, both MAVs were out of the 
other Safe Zone, which makes the collision avoidance module inform the rest of the system that now the quadcopter can return to its original final waypoint.

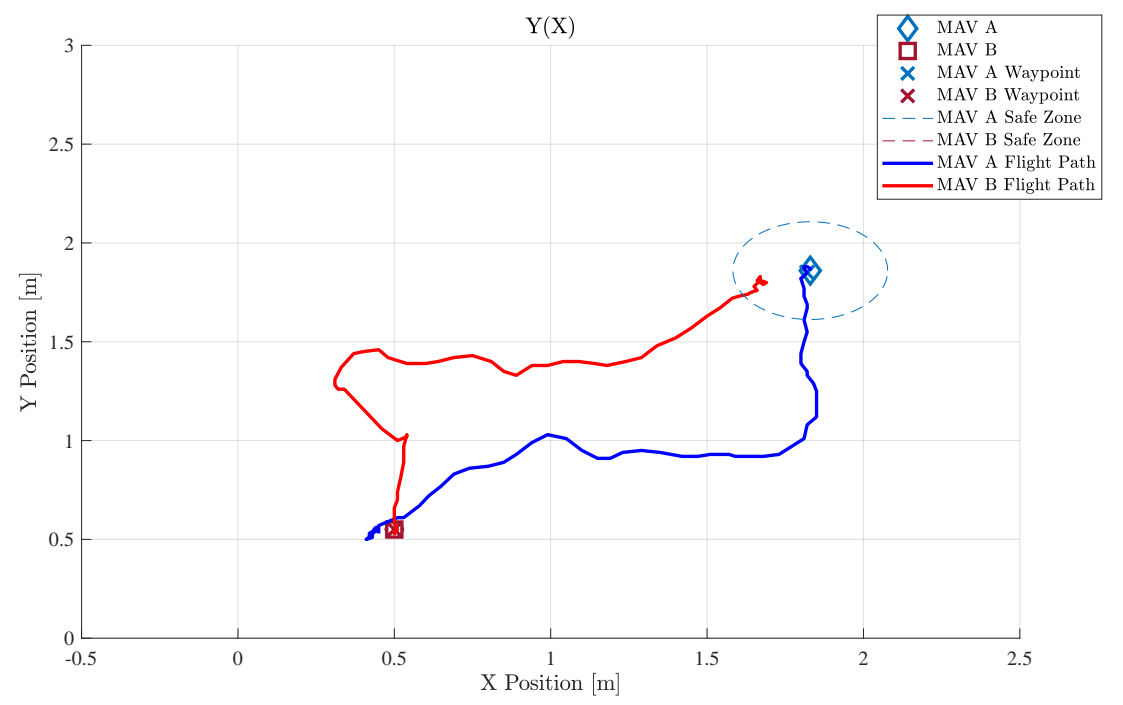

Figure 5.5: Final trajectories and avoidance of head-on collision

Figure 5.5 shows that after the completed avoidance maneuver, the MAVs head for their respective original setpoints, resuming the original flight plans. Note the evasive waypoints have disappeared since the collision situation no longer exists.

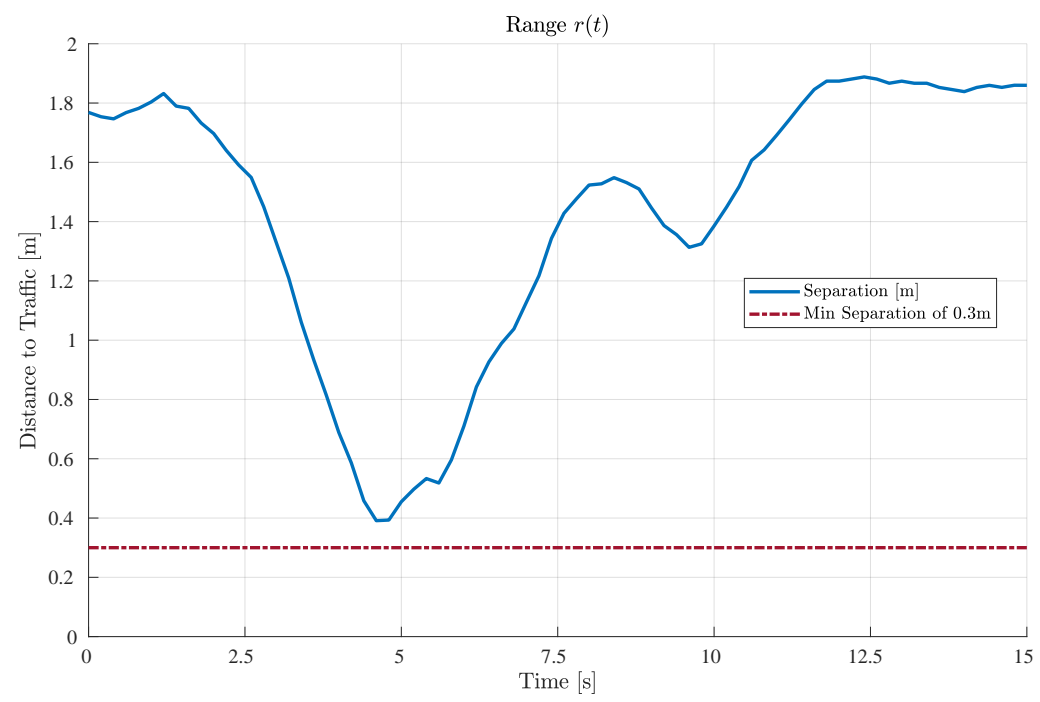

Figure 5.6: Separation between the MAVs during the head-on collision scenario

Figure 5.6 represents the separation distance between the MAVs throughout the flight. Note the separation distance does not fall below the minimum threshold of $0.3[\mathrm{~m}]$. A collision between two MAVs has been prevented while adhering to Rules of the Air outlined by ICAO. 

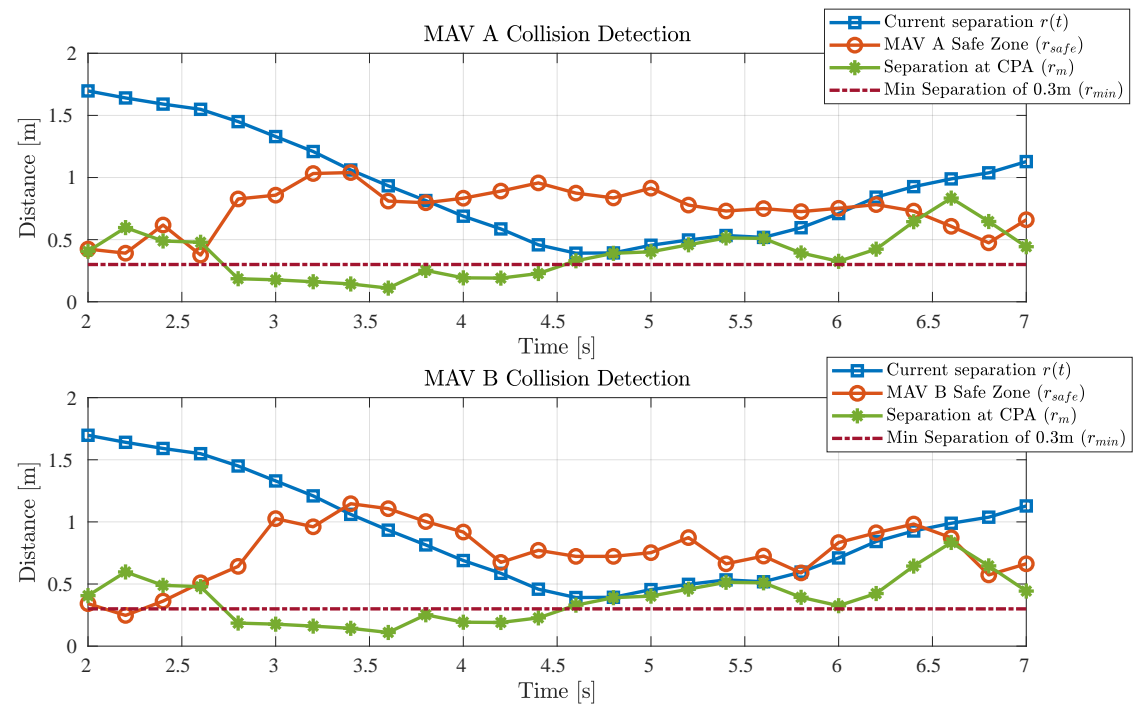

Figure 5.7: Collision detection analysis for head-on scenario

Figure 5.7 represents the evolution over time of the variables that are used to detect a collision scenario. The analysis of these graphs shows that MAV A detects a collision at $t=4 \mathrm{~s}$ as the current separation is decreasing (MAVs are approaching each other), the safe zone size $r_{\text {safe }}$ is greater than the separation distance $r(t)$, and the calculated separation distance at the CPA is less than the minimum threshold of $0.3 \mathrm{~m}$. The same analysis can be done for MAV B, but the detection is triggered a little earlier at $t=3.4 \mathrm{~s}$.
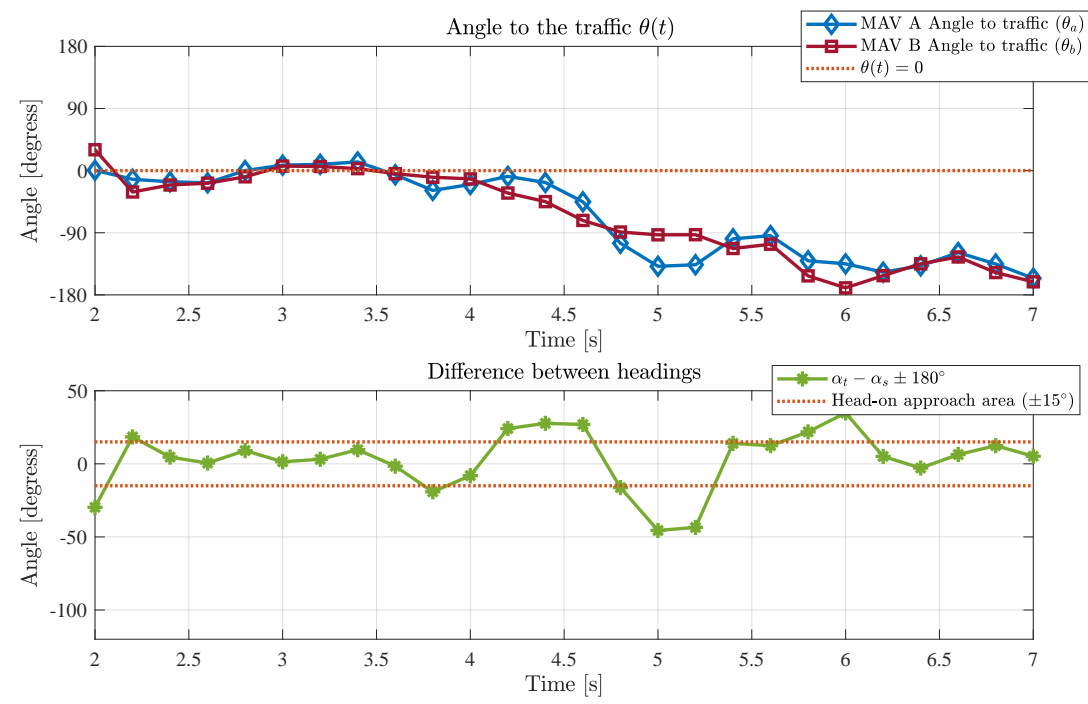

Figure 5.8: Identification of head-on collision scenario

The system tries to reach the evasive waypoint until the intruder is out of the quadcopter respective safe zone, i.e $r(t)>r_{\text {safe }}$. MAV A detects that it is out of danger at $t=6.2 \mathrm{~s}$. The system 
changes to its initial flight path as the conditions for a collision are never met again. The same occurs for MAV B at $t=5.8 \mathrm{~s}$, but now it can be seen that $r(t)<r_{\text {safe }}$ in the next 3 measurements. This did not trigger the collision flag as $r(t)$ were increasing (quadcopters were moving away) and $\left\|r_{m}\right\|>r_{\text {min }}$.

The head-on collision scenario was identified as shown in figure 5.8. It is easy to visualize that for $t<4 \mathrm{~s}$ the difference between the MAVs headings is most of the time inside the area of $\pm 15^{\circ}$ that identifies a head-on collision scenario. As the quadcopters start the avoidance maneuvers, their angles to the traffic changes following the new heading that they take (traffic remains on the left for $t>3.6 \mathrm{~s})$.

\subsubsection{Close approach}

After the end of the experiment of the head-on collision, the quadcopters have to be re-positioned to simulate the converging collision scenario. As showed in figure 5.9 MAV A starts at $\vec{p}_{a}=$ $(1.82,1.85,0.84)$ and head to $\vec{p}_{\text {goal }_{a}}=(0.50,0.55,0.84)$. MAV B starts at $\vec{p}_{b}=(0.50,0.55,0.84)$ and head to $\vec{p}_{\text {goal }_{b}}=(0.50,1.85,0.84)$.

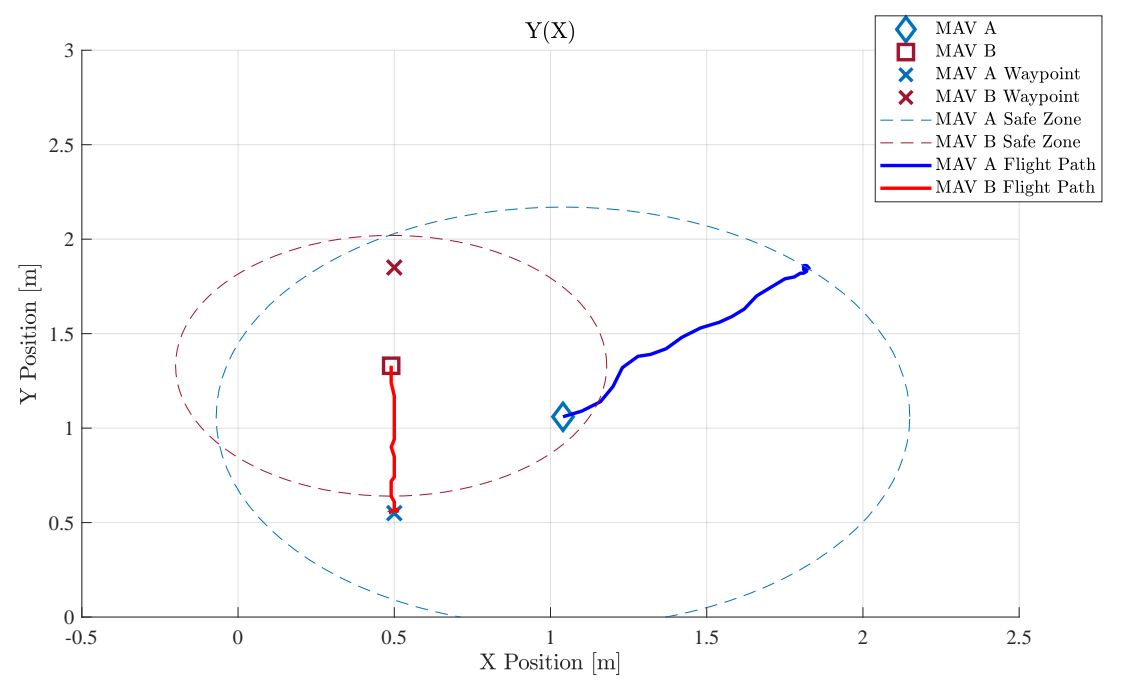

Figure 5.9: MAVs inside each other safe zone in the close approach scenario

Note that at the moment represented in figure 5.9 both MAVs are inside the safe zone of the other and getting closer. However, the minimum separation distance is never violated by the predicted separation at CPA at the critical moments.

It can be seen in figure 5.10 at $t=3.8 \mathrm{~s}$ that MAV A detected traffic inside its safe zone. The same occurs for MAV B at $t=4.6 \mathrm{~s}$, the moment where both quadcopters are approaching and have a safe zone $r_{\text {safe }}$ that is greater than the current separation distance $r(t)$. The quadcopters does not start any evasive maneuver as $\left\|\overrightarrow{r_{m}}\right\|$ is always greater than the minimum separation of $0.3 \mathrm{~m}$. This experiment proved the effectiveness to predict the separation distance at CPA and use it as a condition to detect a collision scenario. 


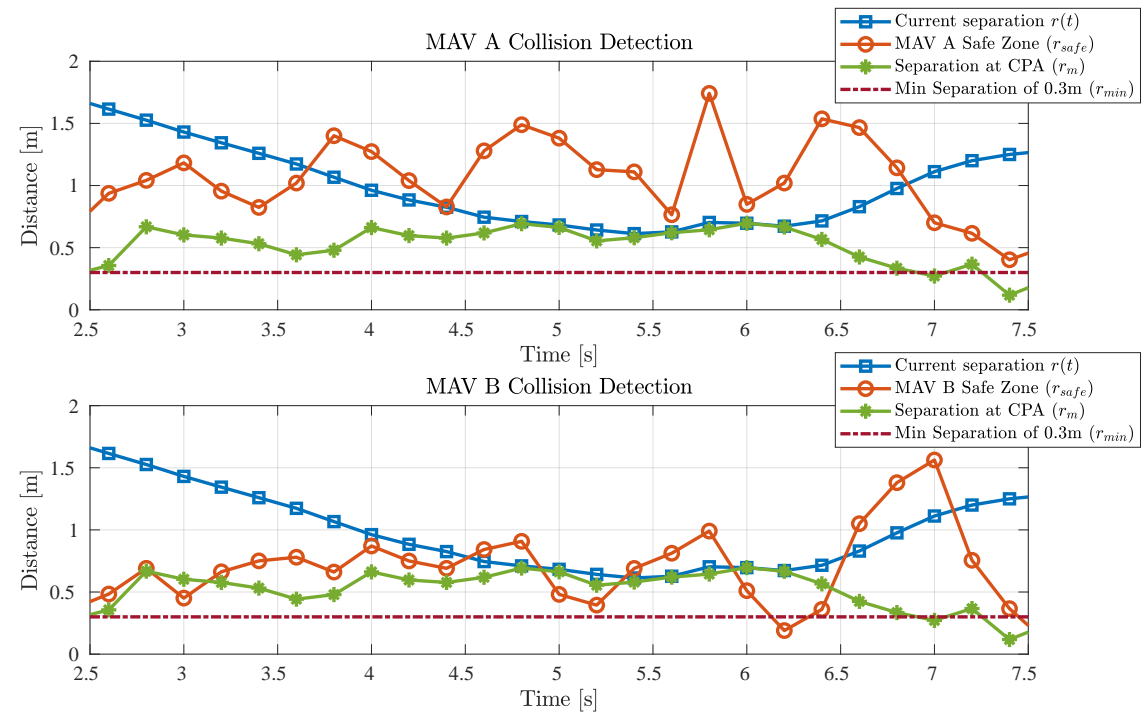

Figure 5.10: Collision detection analysis for close approach scenario

\subsubsection{Converging collision}

As the quadcopters are already positioned after the close approach experiment, the computer commands each one of them to a new setpoint, $\vec{p}_{\text {goal }_{a}}=(1.82,1.85,0.84)$ for MAV A and $\vec{p}_{\text {goal }_{b}}=$ $(1.82,0.55,0.84)$ for MAV $\mathrm{B}$, to force a converging collision scenario.

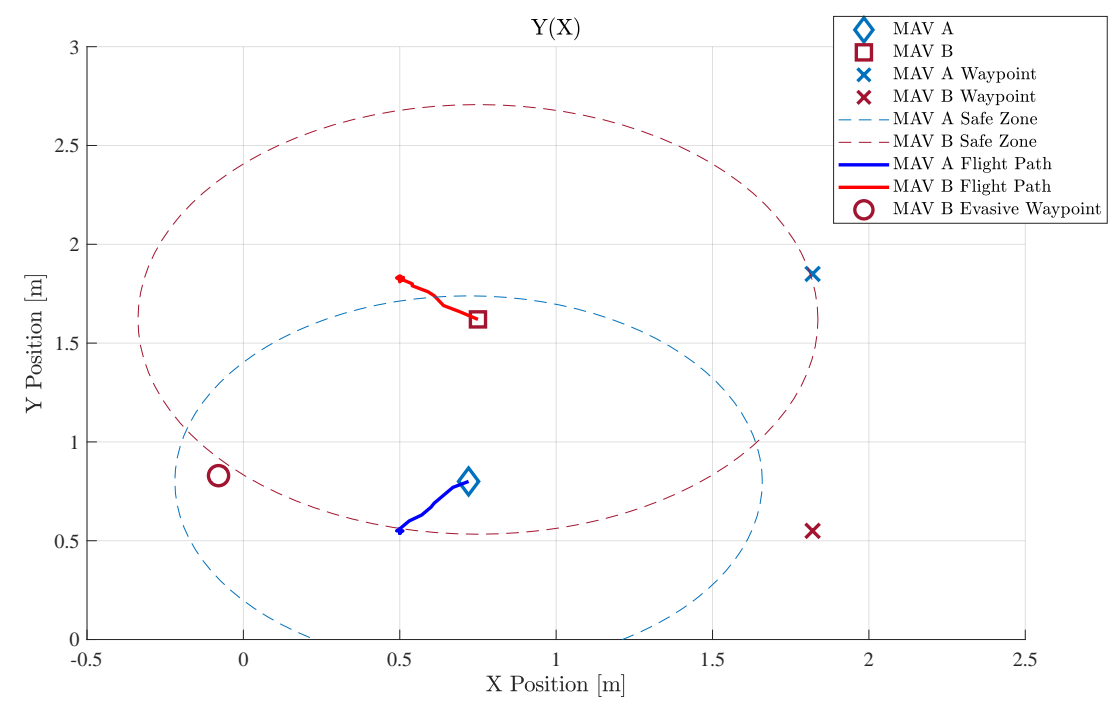

Figure 5.11: Initial detection and beginning of evasive maneuver of converging collision

Figure 5.11 shows a collision situation about to occur due to converging traffic. MAV B has detected MAV A and created an evasive waypoint to head towards, thus attempting to resolve the conflict of collision. 


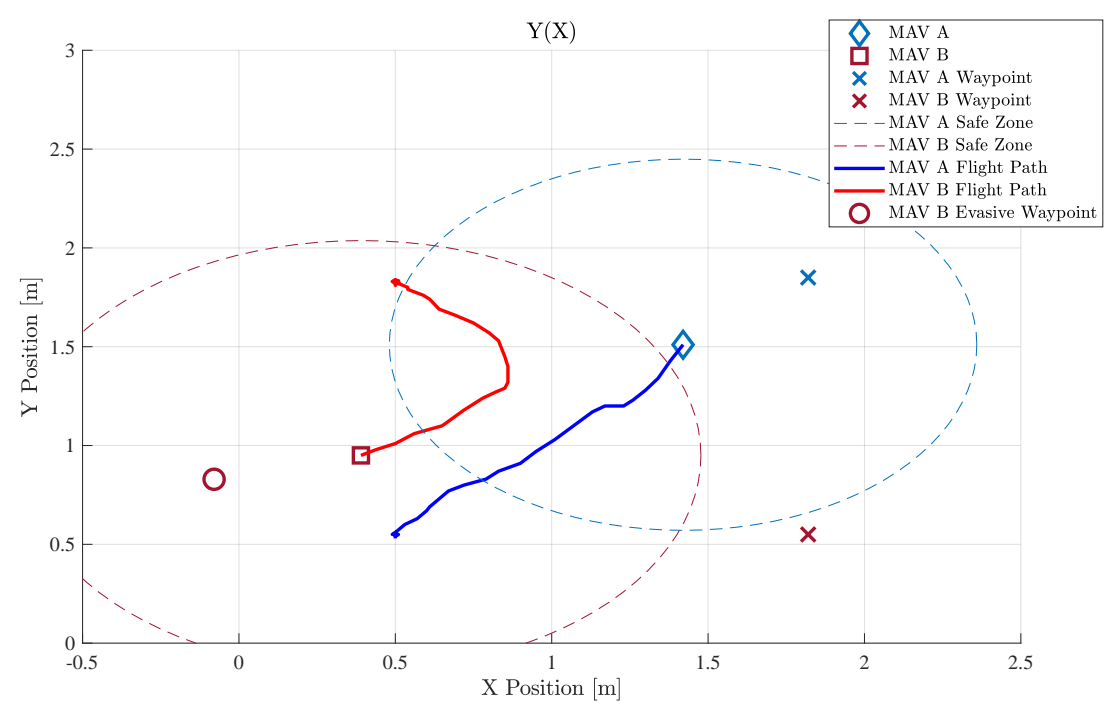

Figure 5.12: Evasive maneuver of the converging traffic collision scenario

Figure 5.12 shows the moment where traffic convergence has been resolved. It can be seen that only MAV B has executed an evasive maneuver and resolved the potential collision conflict. This is as required since MAV B is on the left of MAV A as it does not need to maneuver to evade the collision and still adheres to the rules.

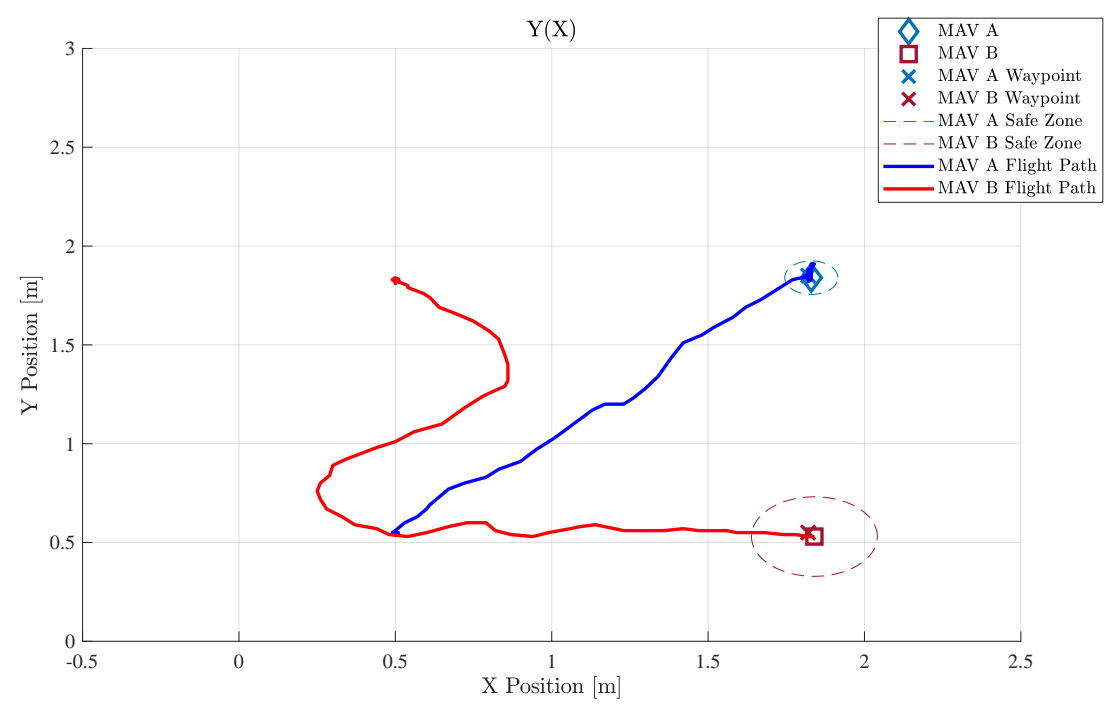

Figure 5.13: Final trajectories and avoidance of converging traffic collision

In figure 5.13 it can be seen that the conflict has been resolved and MAV B resumes its original flight plan. Note the evasive waypoint has disappeared since the collision situation no longer exists. 


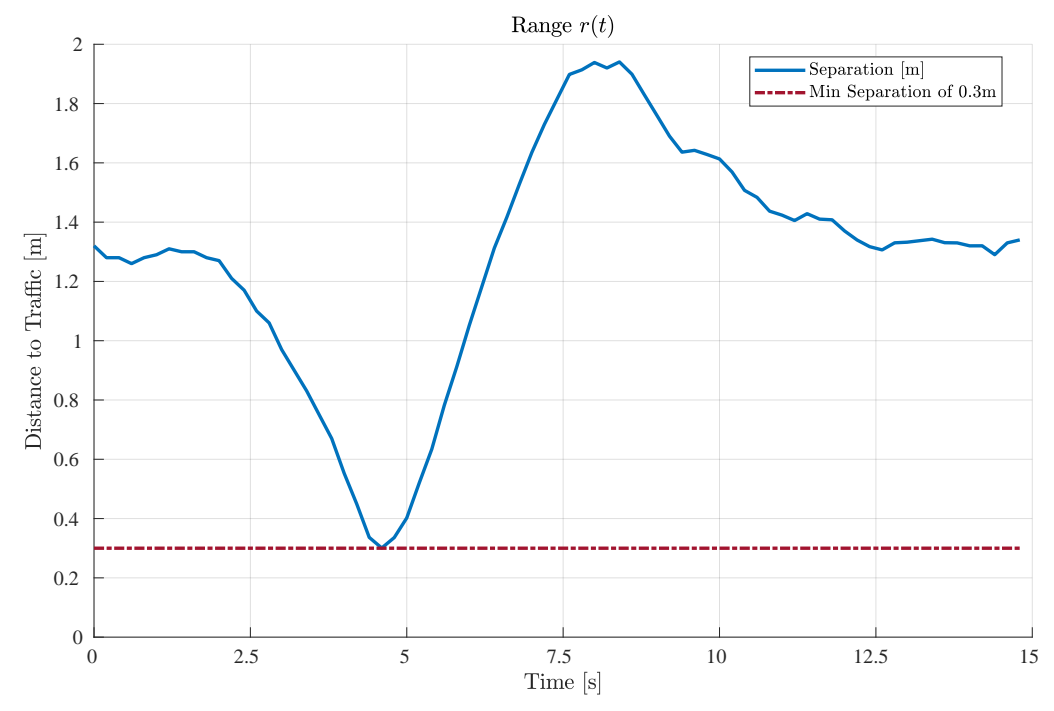

Figure 5.14: Separation between the MAVs during the converging traffic collision scenario

Figure 5.14 represents the separation between the quadcopters throughout the experiment. The separation does not fall below the minimum separation threshold of $0.3 \mathrm{~m}$ again. This confirms collision avoidance for converging traffic functions successfully whilst adhering to Visual Flight Rules.

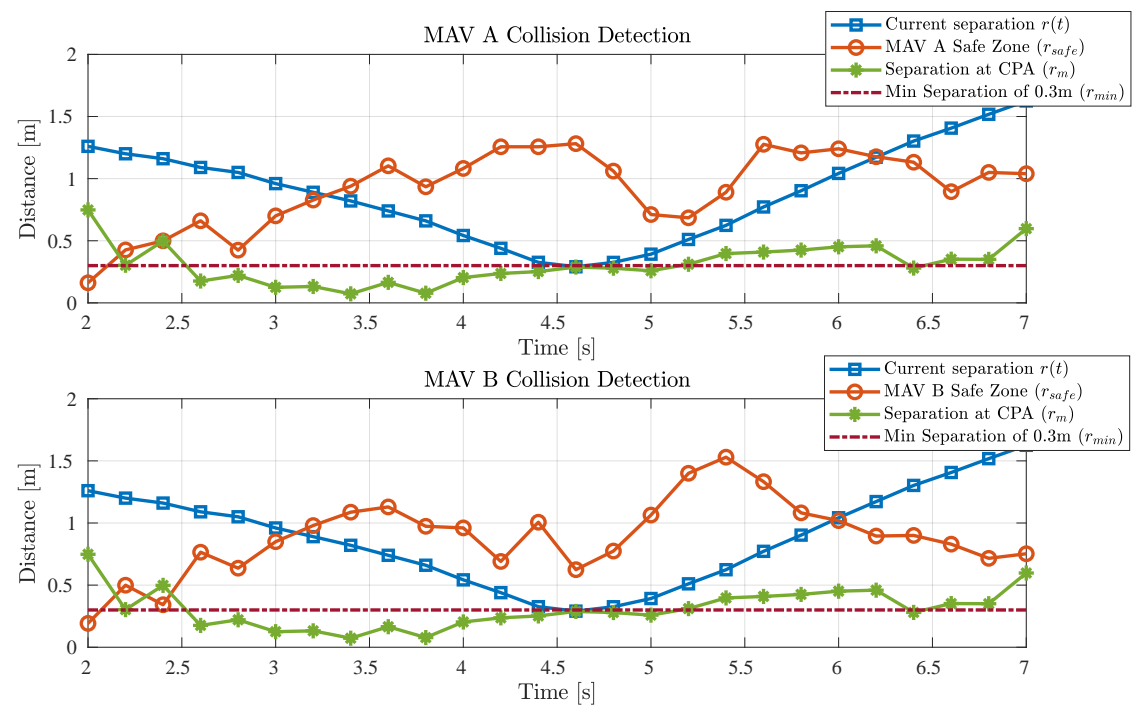

Figure 5.15: Collision detection analysis for converging traffic scenario

Figure 5.15 representation of the variables that are used to detect a collision scenario in critical moments. The analysis of these graphs shows that MAV A detects a collision at $t=3.4 \mathrm{~s}$ as the current separation distance curve $r(t)$ is decreasing, $r_{\text {safe }}>r(t)$, and $\left\|\overrightarrow{r_{m}}\right\|<r_{\min }$. The same analysis can be done for the detection of a collision scenario for MAV B at $t=3.2 \mathrm{~s}$. 
The previous graphs showed that, in the converging traffic scenario, only MAV B had to make the avoidance maneuver, as MAV A continued its normal trajectory. MAV B tries to reach the evasive waypoint until the intruder is out of its safe zone, i.e $r(t)>r_{\text {safe }}$. This happens at $t=6 \mathrm{~s}$ with MAV B changing to its initial flight path as the conditions for a collision are never met again. On the other hand, despite that MAV A have the intruder inside its safe zone until $t=6.2 \mathrm{~s}$, it continues to its original waypoint as required by the Rules of the Air standard.
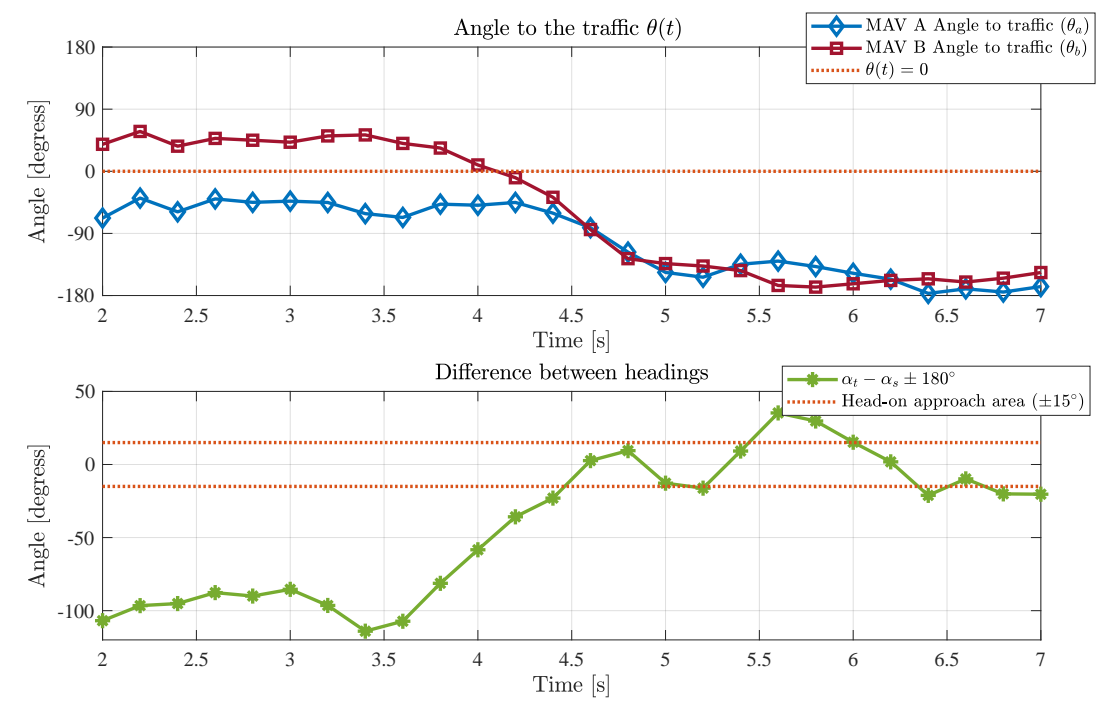

Figure 5.16: Identification of converging traffic collision scenario

The converging traffic collision scenario was identified as shown in figure 5.16. The Angle to the traffic graph shows that when the collision was detected by MAV B at $t=3.2 \mathrm{~s}$, it had the traffic on its right, i.e. $\theta_{b}>0^{\circ}$. That was the condition that makes MAV B starts the avoidance maneuver. On the other hand, MAV A always had the traffic on its left, i.e. $\theta_{a}<0^{\circ}$, what was the condition for it to maintain its original flight path. In contrast with the behavior of the head-on scenario, the difference between the quadcopters headings before the detection is far from the $\pm 15^{\circ}$ area. This is due to the clear distinct behaviors that both scenarios provide to the collision avoidance module.

\subsection{Conclusions}

The analysis of the results presented in this chapter can be used to highlight a few conclusions for the proposed collision avoidance system.

- Treat the virtual force of the APF as the force that acts on the quadcopter to create acceleration (Newton's $2^{\circ}$ law) had some problems with the original APF approach. The addition of the error for the desired velocity term on the attractive force equation solved the oscillatory motion problem. 
- Despite the problems regarding the radio chip that was used for P2P communication, the approach resulted in a useful way to adapt the ADS-B technology. The lower average rate of received $\mathrm{P} 2 \mathrm{P}$ packets did not influence the overall effectiveness of the detection and avoidance methods.

- The usage of three metrics to predict a collision scenario had credible results. As the detection was tested for three different scenarios, the experiments showed the robustness of a simple flight plan sharing approach with a kinematic system modeling.

- The approach used to define the criteria for each collision scenario (head-on and converging traffic) was a reliable way to determine which quadcopter should perform the avoidance maneuver. The results also showed that the envelope of $\pm 15^{\circ}$ was enough to detect the possible head-on collision, in agreement with the literature.

- The hybrid approach used for the collision avoidance maneuvers proved to be effective in ensuring safe motion for the quadcopters. The evasive waypoint created a safe path for the conflict MAVs as the APF guided the quadcopters through the environment with controlled velocity and smoother trajectories. 


\section{Chapter 6}

\section{Conclusions}

As autonomy and collision avoidance technologies improve, more research is conducted to increase the ability of Unmanned Aerial Vehicles to perform complex tasks. The emergence of new ways of private transportation like eVTOL is a reality. Regulations and standards regarding the operation of these vehicles will be needed soon. Taking ideas from systems already well established are some times good ways to construct and adopt new technologies to the world. For the eVTOLs, it will probably be no different.

This dissertation was intended to study, adapt, and validate technologies and methods of collision avoidance systems currently used in aeronautics to the use-case of VTOL UAVs. Here, the three technological areas that compose the sense and avoid paradigms were addressed, and a complete functional collision avoidance system was implemented into micro indoor quadcopters for proof of concept. A cooperative-traffic detection was obtained by adapting the ADS-B technology into the low-cost MAVs. The proposed collision detection and risk evaluation methods are parameterized using the traffic data from the ADS-B and a geometric approach from a simple kinematic model that predicts if a collision is imminent or not. Finally, to perform safe maneuvers, the system uses an evasive waypoint to avoid the traffic and a Potential Field method to control the speed and smooth the motion trajectories.

The developed system was tested with both simulation and real-world experiments. The use of real hardware experiments promoted the validation of the proposed methods and algorithms, as requested by the Altran Portugal R\&D team. During the development of this work, the project was presented on several workshops and live demos that could show for possible future partnerships the technological progress of Altran PT in the UAM field. Despite that some adaptations and modifications had to be made in the original approaches to make the whole system suitable for the MAVs used for real-world experiments, the solution showed promising results regarding the robustness, fast computation, and low processing power needed.

In the end, the objectives that were in the scope of this dissertation were all successfully achieved. The main challenge lied in the usage of real-world systems and tools to develop a full prototype of the proposed collision avoidance methods and technologies. However, as the realworld results showed, this solution granted to the MAVs the ability to sense and detect conflict 
traffic and perform standardized avoidance maneuvers while adhering to the Rules of the Air VFR conditions and ICAO requirements.

\subsection{Future Work}

This work could benefit from future research on some interesting topics:

- With the available hardware, this work only implemented detection for cooperative traffic. The addition of cameras or infrared sensors could enable the collision avoidance maneuvers for non-cooperative traffic such as other UAVs that do not dispose of an ADS-B transponder or even distractors, such as birds.

- As this work adapts the ADS-B behavior in the MAV radio chip over $2.4 \mathrm{GHz}$, future adoption of a real ADS-B transponder could be a valuable addition to the system. That could enable tests regarding the distance of communication and the number of quadcopters that can share the same channel without a critical interference to the collision avoidance system.

- Despite that the simple kinematic model used to predict a probable collision of the MAVs was enough for the scope of this dissertation, the addition of a probabilistic model could increase the effectiveness of the detection methods. By including some uncertainties, the developed system could be extended to detect and perform better avoidance maneuvers given a specific scenario.

- The series of experiments of this dissertation only consider the collision scenarios between two MAVs. If multiple quadcopters are to be avoided, that can be done by calculating evasive waypoints for each one of them and start the maneuver towards the one with the largest bearing.

- There is one more conflict situation that could lead to a collision scenario, the overtaking situation as defined by the ICAO Rules of the Air. The developed architecture could be extended to incorporate the detection and avoidance of that last conflict scenario. 


\section{References}

[1] SESAR Joint Undertaking. European Drones Outlook Study. Technical report, European Commission, November 2016.

[2] CNN. What's more fancy than a Porsche? A flying Porsche. Luxury automakers race to perfect the flying car. Available at https://edition.cnn.com/travel/article/ flying-cars-automakers/index.html, last accessed on September 14, 2020.

[3] Uber. Uber Air. Available at https://www.uber.com/us/en/elevate/uberair/, last accessed on September 14, 2020.

[4] Airbus. Urban Air Mobility. Available at https://www.airbus.com/innovation/ zero-emission/urban-air-mobility.html, last accessed on September 14, 2020.

[5] Aurora Flight Sciences. PAV - eVTOL Passenger Air Vehicle. Available at https : / / www . aurora.aero/pav-evtol-passenger-air-vehicle/, last accessed on September $14,2020$.

[6] Rolls-Royce. Electric propulsion for a disruptive mobility concept. Available at https: //www.rolls-royce.com/innovation/propulsion/air-taxis.aspx, last accessed on September 14, 2020.

[7] Uber Elevate. Uber Air Vehicle Requirements and Missions. Technical report, Uber, March 2018.

[8] Altran. En-Route to Urban Air Mobility: On The Fast Track To Viable and Safe On-Demand Air Services. Technical report, Altran, 2020.

[9] Federal Aviation Administration. Integration of Civil Unmanned Aircraft Systems (UAS) in the National Airspace System (NAS) Roadmap. Technical report, FAA, July 2018.

[10] Laith Sahawneh. Airborne Collision Detection and Avoidance for Small UAS Sense and Avoid System. PhD thesis, Brigham Young University, 2016.

[11] International Civil Aviation Organization. Annex 2 to the Convention on International Civil Aviation - Rules of the Air. ICAO, Tenth edition, 2005.

[12] Jovan Boskovic, Joseph Jackson, and Raman Mehra. Sensor and Tracker Requirements Development for Sense and Avoid Systems for Unmanned Aerial Vehicles. In AIAA Modeling and Simulation Technologies (MST) Conference, 2013.

[13] James Mackie. Compact FMCW Radar for GPS-Denied Navigation and Sense and Avoid. Master's thesis, Brigham Young University, 2014. 
[14] John Lai, Jason Ford, Luis Mejias Alvarez, Peter O'Shea, and Rodney Walker. See and avoid using onboard computer vision. In A Plamen, editor, Sense and Avoid in UAS: Research and Applications, pages 265-294. John Wiley \& Sons, USA, 2012.

[15] L. Zheng, P. Zhang, J. Tan, and F. Li. The Obstacle Detection Method of UAV Based on 2D Lidar. IEEE Access, 7:163437-163448, 2019.

[16] Cláudia Vanessa Coutinho Rodrigues. ADS-B - Automatic Dependent SurveillanceBroadcast - estudo do impacto em Portugal. Master's thesis, Universidade da Beira Interior, 2010.

[17] International Civil Aviation Organization. Doc 4444, Procedures for Air Navigation Services - Air Traffic Management. ICAO, Sixteenth edition, 2016.

[18] Franco Minucci, Evgenii Vinogradov, and Sofie Pollin. Avoiding Collisions at Any (Low) Cost: ADS-B Like Position Broadcast for UAVs. IEEE Access, 8:121843-121857, 2020. URL: http://dx.doi.org/10.1109/ACCESS.2020.3007315, doi:10. $1109 /$ access.2020.3007315.

[19] Y. Pan, S. Li, and B. Li. When UAVs coexist with manned airplanes: Large-scale aerial network management using ADS-B. In Transactions on Emerging Telecommunications Technologies, 2019.

[20] B. M. Albaker and N. A. Rahim. Unmanned aircraft collision detection and resolution: Concept and survey. In 2010 5th IEEE Conference on Industrial Electronics and Applications, pages 248-253, 2010.

[21] M. P. Owen, A. Panken, R. Moss, L. Alvarez, and C. Leeper. ACAS Xu: Integrated Collision Avoidance and Detect and Avoid Capability for UAS. In 2019 IEEE/AIAA 38th Digital Avionics Systems Conference (DASC), pages 1-10, 2019.

[22] Baraa Albaker and Nasrudin Abd Rahim. Unmanned aircraft collision avoidance system using cooperative agent-based negotiation approach. International Journal of Simulation: Systems, Science and Technology, 11:01-07, 092010.

[23] Hamid Alturbeh and James F. Whidborne. Visual Flight Rules-Based Collision Avoidance Systems for UAV Flying in Civil Aerospace. Robotics, 9:9, 2020.

[24] A. Budiyanto, A. Cahyadi, T. B. Adji, and O. Wahyunggoro. UAV obstacle avoidance using potential field under dynamic environment. In 2015 International Conference on Control, Electronics, Renewable Energy and Communications (ICCEREC), pages 187-192, 2015.

[25] Y. Lin and S. Saripalli. Sampling-Based Path Planning for UAV Collision Avoidance. IEEE Transactions on Intelligent Transportation Systems, 18(11):3179-3192, 2017.

[26] M. Shanmugavel, A. Tsourdos, and B. A. White. Collision avoidance and path planning of multiple UAVs using flyable paths in 3D. In 2010 15th International Conference on Methods and Models in Automation and Robotics, pages 218-222, 2010.

[27] Bitcraze AB. Bitcraze Official Website. Available at https://www.bitcraze.io/, last accessed on September 17, 2020.

[28] Bitcraze AB. Bitcraze GitHub account. Available at https://github.com/bitcraze/, last accessed on September 14, 2020. 
[29] M. Fabrice Poirier. Control of the quadcopter Crazyflie. Technical report, University of Manchester, September 2018.

[30] M. Greiff. Modelling and Control of the Crazyflie Quadrotor for Aggressive and Autonomous Flight by Optical Flow Driven State Estimation. Master's thesis, Dept of Automatic Control, Lund University, 2017.

[31] Bitcraze AB. Crazyflie Firmware. Bitcraze GitHub repository. Available at https:// github.com/bitcraze/crazyflie-firmware/tree/master/src, last accessed on September 17, 2020.

[32] Raffaello D'Andrea. Lectures of Recursive Estimation. ETH Zurich. Available at https : / / idsc.ethz.ch/education/lectures/recursive-estimation.html, last accessed on September 14, 2020.

[33] J. A. Preiss, W. Honig, G. S. Sukhatme, and N. Ayanian. Crazyswarm: A large nanoquadcopter swarm. In 2017 IEEE International Conference on Robotics and Automation (ICRA), pages 3299-3304, 2017.

[34] D. Mellinger and V. Kumar. Minimum snap trajectory generation and control for quadrotors. In 2011 IEEE International Conference on Robotics and Automation, pages 2520-2525, 2011.

[35] Ewoud Smeur, Q. Chu, and Guido Croon. Adaptive Incremental Nonlinear Dynamic Inversion for Attitude Control of Micro Aerial Vehicles. In Journal of Guidance, Control, and Dynamics: devoted to the technology of dynamics and control, 012016. doi: $10.2514 / 6.2016-1390$.

[36] Bitcraze AB. Getting started with the Loco Positioning system. Bitcraze Tutorials. Available at https://www.bitcraze.io/documentation/tutorials/ getting-started-with-loco-positioning-system/, last accessed on September 17, 2020.

[37] Bitcraze AB. Crazyflie Python Library. Bitcraze GitHub repository. Available at https: //github.com/bitcraze/crazyflie-lib-python, last accessed on June 7, 2020.

[38] Bitcraze AB. Crazyflie 2.0 Model. Bitcraze GitHub repository. Available at https:// github.com/bitcraze/bitcraze-mechanics/tree/master/models, last accessed on September 15, 2020.

[39] Julian Förster. System Identification of the Crazyflie 2.0 Nano Quadcopter. Master's thesis, Institute for Dynamic Systems and Control Swiss Federal Institute of Technology, Zurich, Switzerland, 2015.

[40] Fadri Furrer, Michael Burri, Markus Achtelik, and Roland Siegwart. Robot Operating System (ROS): The Complete Reference (Volume 1), chapter RotorS-A Modular Gazebo MAV Simulator Framework, pages 595-625. Springer International Publishing, Cham, 2016. URL: http://dx.doi.org/10.1007/978-3-319-26054-9_23, doi:10. 1007/978-3-319-26054-9_23.

[41] Crazyflie Community. Gazebo Simulation for Crazyflie CRTP. GitHub repository. Available at https://github.com/wuwushrek/sim_cf, last accessed on September 17, 2020. 
[42] R. Schollmeier. A definition of peer-to-peer networking for the classification of peer-to-peer architectures and applications. In Proceedings First International Conference on Peer-toPeer Computing, pages 101-102, 2001.

[43] César Muñoz, Anthony Narkawicz, and James Chamberlain. A TCAS-II resolution advisory detection algorithm. In AIAA Guidance, Navigation, and Control (GNC) Conference, 08 2013. doi:10.2514/6.2013-4622.

[44] C. Mishra, M. Mehta, E. J. Griffith, and J. F. Ralph. Doing the Right Thing: Collision Avoidance for Autonomous Air Vehicles. In 2013 IEEE International Conference on Systems, Man, and Cybernetics, pages 2581-2586, 2013.

[45] M. Isabel Ribeiro. Obstacle Avoidance. Pedagogical Publications. Instituto de Sistemas e Robótica, Instituto Superior Técnico, November 2005.

[46] Veysel Gazi and Kevin M. Passino. Swarms of Double Integrator Agents, pages 75-105. Springer Berlin Heidelberg, Berlin, Heidelberg, 2011. URL: https://doi.org/10 . 1007/978-3-642-18041-5_4, doi:10.1007/978-3-642-18041-5_4. 